This is the final peer-reviewed accepted manuscript of:

Paracampo, R., Pirruccio, M., Costa, M., Borgomaneri, S., \& Avenanti, A. (2018). Visual, sensorimotor and cognitive routes to understanding others' enjoyment: An individual differences rTMS approach to empathic accuracy. Neuropsychologia, $116,86-98$.

\begin{tabular}{|lllll}
\hline The final published version is & available & online & at: \\
https://doi.org/10.1016/j.neuropsychologia.2018.01.043 & & \\
\hline
\end{tabular}

Rights / License:

The terms and conditions for the reuse of this version of the manuscript are specified in the publishing policy. For all terms of use and more information see the publisher's website.

\title{
Visual, sensorimotor and cognitive routes to understanding others' enjoyment: An individual differences rTMS approach to empathic accuracy
}

Riccardo Paracampo ${ }^{1,2, \#, \text { Martina Pirruccio }}{ }^{2}$, Marco Costa ${ }^{3}$, Sara Borgomaneri², Alessio Avenanti ${ }^{1,2, *}$

\footnotetext{
${ }^{1}$ IRCCS Fondazione Santa Lucia, 00179 Rome, Italy; ${ }^{2}$ Department of Psychology, University of Bologna, and Center for Studies and Research in Cognitive Neuroscience, University of Bologna, 47521 Cesena, Italy;

${ }^{3}$ Department of Psychology, University of Bologna, 40127 Bologna, Italy.
}

\# Now at Netherlands Institute for Neuroscience

* Correspondence should be addressed to: Alessio Avenanti. Centro studi e ricerche in Neuroscienze

Cognitive, Alma Mater Studiorum Università di Bologna, Campus di Cesena. VialeEuropa 980, 47521

Cesena, Italy. Voice: +390547338951, Facsimile:+390547338952. E-mail: alessio.avenanti@unibo.it 


\begin{abstract}
Functional imaging studies suggest that accurate understanding of others' emotional feelings (i.e., empathic accuracy, EA) recruits high-order visual, sensorimotor and mentalizing brain networks. However, the behavioral relevance of these findings is unclear. To fill in this gap, we used repetitive transcranial magnetic stimulation (rTMS) to interfere with the right superior temporal sulcus (STS), inferior frontal gyrus (IFG) and temporoparietal junction (TPJ) during an EA task requiring participants to infer the enjoyment felt by a social target while smiling/laughing. Relative to a baseline condition (sham rTMS), active rTMS of STS, IFG and TPJ (but not of a control site) disrupted the efficiency of EA task performance, mainly by lowering task accuracy; rTMS of IFG and TPJ also slowed down response speeds. Importantly, the effects of rTMS on EA task efficiency were predicted by baseline EA performance, with high-performers showing a performance decrease when the TPJ was targeted, and low-performers showing a performance decrease when the STS or the IFG was targeted. The double dissociation in the effect of rTMS between low- and highperformers suggests distinct roles of STS, IFG and TPJ in efficient understanding of the enjoyment felt by others. These findings provide causal evidence of distinct visual, sensorimotor and cognitive routes to EA and suggest that individual differences in EA are underpinned by differential recruitment of these routes.
\end{abstract}

\title{
Graphical abstract
}

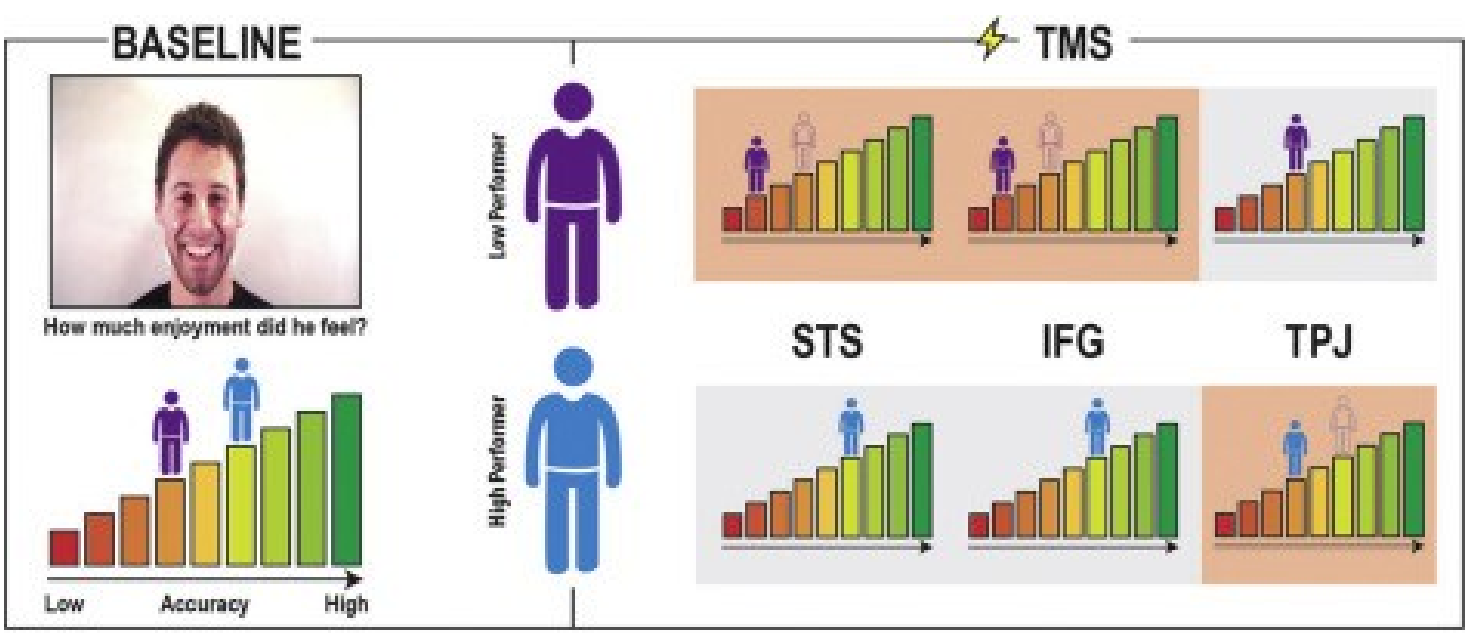

Keywords: Empathic accuracy; Emotion recognition; Individual differences; Transcranial magnetic stimulation; Superior temporal sulcus (STS); Inferior frontal gyrus (IFG);

Temporoparietal junction (TPJ) 


\section{Highlights}

- rTMS over STS, IFG and TPJ impaired empathic accuracy (EA).

- Individual differences in EA moderated the effects of rTMS.

- Low EA-performers showed impaired EA following rTMS over STS and IFG.

- High EA-performers showed impaired EA following rTMS over TPJ.

- Low and high EA-performers differentially recruited the brain regions supporting EA.

\section{Introduction}

Among all the facial expressions we encounter during social interactions, the smiling face is one of the most common and yet one of the most ambiguous (Niedenthal et al., 2010). Smiles and laughter are considered readouts of felt enjoyment and are essential for the creation and maintenance of social bonds (Cashdan, 1998, Fridlund, 2002). Thus, being able to accurately understand the enjoyment behind a smile/laughter is critical for social life.

The ability to accurately infer the internal emotional state felt by another individual (a social target) is often referred to as empathic accuracy (EA) (Ickes and Stinson, 1990, Levenson and Ruef, 1992, Ickes, 1997, Zaki et al., 2008, Zaki et al., 2009a) and it is defined as the correspondence between the feelings reported by the social target and the feelings that perceivers infer from the social target's behavior.

The chain of neuropsychological processes leading to EA starts with visual processing of morphological and dynamic features of the social target's expression (Ambadar et al., 2009, Vuilleumier and Huang, 2009, Pourtois et al., 2013, Calvo and Nummenmaa, 2016). A key brain area involved in processing facial expressions is the posterior sector of the superior temporal sulcus (STS) (Haxby et al., 2000, Winston et al., 2003, Winston et al., 2004, Sabatinelli et al., 2011, Harris et al., 2012, Saarimäki et al., 2016), which is a multisensory region showing sensitivity to several non-verbal social signs including biological motion, actions, gaze and vocalizations (Allison et al., 2000, Jellema and Perrett, 2006, Nummenmaa and Calder, 2009, Carlin and Calder, 2013).

In addition to visual processes, further sensorimotor and cognitive processes might support EA, including sensorimotor simulation and mentalizing (Zaki et al., 2012b, Wood et al., 2016, Paracampo et al., 2017). The former is a process by which a viewer partially reproduces the social target's facial expression in their own sensorimotor system. Sensorimotor simulation is 
thought to contribute to emotion recognition (Goldman and Sripada, 2005; Oberman et al., 2007; Korb et al., 2010, Korb et al., 2014; Rychlowska et al., 2014) via recruitment of a set of somatosensory, motor and premotor areas (Niedenthal et al., 2010, Gallese and Sinigaglia, 2011; Korb et al., 2015; Wood et al., 2016; Borgomaneri et al., 2012), including the inferior frontal gyrus (IFG), a rostral motor region involved in controlling facial movements and linking action to cognition (Carr et al., 2003, Dapretto et al., 2006, Sabatinelli et al., 2011, Rizzolatti et al., 2014). Mentalizing processes allow the viewer to explicitly consider others' mental states and their sources, and to draw explicit inferences about them (Amodio and Frith, 2006, Frith and Frith, 2006, Mitchell, 2009). These processes might be critical for EA tasks requiring participants to explicitly judge others' internal emotional states (Zaki et al., 2009b, Zaki et al., 2012b). Studies exploring mental state attributions highlighted a subset of midline and lateral regions of the brain constituting the so-called mentalizing network and including the temporo-parietal junction (TPJ) (Saxe, 2006, Bzdok et al., 2012, Schurz et al., 2014).

Previous studies often investigated visual, sensorimotor and cognitive mechanisms in social cognition by assessing brain activations with either passive viewing tasks involving static or dynamic displays of actions/emotional expressions (typically showing visual and sensorimotor activations encompassing STS and IFG) or explicit inferential tasks based on verbal materials or on abstract or stylized visual cues (typically showing activations mainly in mentalizing areas, including the TPJ) (e.g., Carr et al., 2003; Dapretto et al., 2006; Frith and Frith, 2006; Saxe, 2006; Mitchel1, 2009; Johnston et al., 2013; see Zaki et al., 2012a for an excellent review). Recently, more naturalistic neuroscientific paradigms have been used to investigate EA by combining dynamic social cues (i.e., a social target's expressive behavior) and explicit inferential tasks (i.e., explicit judgments about the social target's underlying emotional state) (Zaki et al., 2009b, Zaki et al., 2012b, Harvey et al., 2013, McGettigan et al., 2015). These studies reported that EA performance is predicted by neural activity in high-order visual regions (i.e., STS), sensorimotor regions involved in moving and controlling the body (e.g., premotor cortices) and higher-order cognitive regions involved in mentalizing. However, these correlational studies have left unanswered the critical question of whether visual, sensorimotor and cognitive networks provide routes to understanding others (i.e., are functionally relevant to social understanding), or merely reflect such understanding (Gallese et al., 2011, Avenanti et al., 2013b, Urgesi et al., 2014, Lamm and Majdandžić, 2015).

To answer this question, in the present study, we used repetitive transcranial magnetic stimulation (rTMS) to perturb STS, IFG and TPJ and test their causal involvement in making efficient inferences of enjoyment from others' smiling/laughing expressions. In two experiments, we applied rTMS while participants performed an EA task adapted from previous psychological and neuroscientific research (Zaki et al., 2008, Zaki et al., 2009b, Paracampo et al., 2017). The EA 
task required participants to watch dynamic movies of smiling/laughing facial expressions and judge the level of enjoyment felt by the observed social target. Accuracy in the EA task (i.e., EA scores) reflected the correspondence between the feelings that perceivers inferred from the social target's nonverbal facial expression and the feelings reported by the social target. Response times (RTs) were also assessed, and speed and accuracy indices of task performance were combined into a global measure of performance efficiency.

In Experiment 1, we perturbed activity in the posterior sector of the right STS, which is recruited during the perception of emotional faces, and the primary visual cortex (V1), which served as an active control site. In Experiment 2, we perturbed activity in the right IFG and the right TPJ sites involved in sensorimotor control of facial movements and in mentalizing processes, respectively. In both Experiment 1 and 2, EA performance was also assessed during sham rTMS to provide a baseline condition. If visual, sensorimotor and mentalizing brain regions are functionally relevant to EA, then rTMS over STS, IFG and TPJ should reduce the efficiency of EA task performance. No reduction in EA was expected when interfering with the control site.

Additionally, based on a growing literature showing that the effect of brain stimulation on behavioral tasks is highly variable across individuals (Jones et al., 2016, Palmer et al., 2016, Avenanti et al., 2017, Valchev et al., 2017) and might depend on a number of factors, including individual differences in the ability to perform the task (Krause and Cohen Kadosh, 2014, Benwell et al., 2015, Painter et al., 2015, Emrich et al., 2017, Penton et al., 2017, Yang and Banissy, 2017), we explored the contribution of individual differences in baseline EA task performance to performance changes induced by active rTMS. This allowed us to establish whether low- and high-performers in our EA task show any differential causal involvement of visual, sensorimotor and mentalizing brain areas in EA.

\section{Materials and methods}

\subsection{Participants}

Fifty-six healthy participants took part in the study. Thirty-two subjects were randomly assigned to one of the two TMS experiments. In each TMS experiment, we targeted two different brain regions: V1 and the right STS in Experiment 1 (16 participants, 8 females, mean age \pm SD: $23.7 \pm$ 1.1), and the right IFG and right TPJ in Experiment 2 (16 participants, 8 females, mean age \pm SD: $23.5 \pm 3.0$ ). Moreover, 24 subjects were tested in a pilot study (12 females, mean age \pm SD: $24.9 \pm$ 1.7) aimed at selecting the stimuli for the TMS experiments. All subjects were right-handed, had normal or corrected-to-normal visual acuity in both eyes, and were naïve to the purposes of the experiment. None of the participants had neurological, psychiatric, or other medical problems or any contraindication to TMS ( 
informed consent. The procedures were approved by the University of Bologna Bioethics Committee and were in accordance with the ethical standards of the 1964 Declaration of Helsinki (World Health Organisation, 2013). No discomfort or adverse effects of TMS were reported by participants or noticed by the experimenter.

\subsection{Stimuli and pilot study}

Stimuli consisted of 90 dynamic movies ( $2 \mathrm{~s}, 60$ frames) presented centrally on a 24 -in. monitor (resolution: $1024 \times 768$; refresh rate: $60 \mathrm{~Hz}$ ), subtending a visual angle of $27^{\circ} \times 21^{\circ}$. Movies depicted 6 individuals ("social targets"; including 3 females and 3 males, aged $24.5 \pm 2.1$ years) who were filmed individually while smiling or laughing. Stimuli from the same actors were collected for a previous study using different tasks and procedures (Paracampo et al., 2017). Each movie started with an initial phase (500 ms, 15 frames) showing a neutral static facial expression and a subsequent phase $(1500 \mathrm{~ms}, 45$ frames) showing smile/laughter onset and development (Fig. 1). The last frame of each video clip contained the apex of the expression. Although smile/laughter offset can also be informative of enjoyment/non enjoyment (Ambadar et al., 2009), the expression offset was excluded to keep the duration of the clip short ( $2 \mathrm{~s}$ ) due to temporal constraints of high-frequency rTMS. As in previous research, we opted for a short rTMS train covering the entire duration of the clip (Tidoni et al., 2013, Paracampo et al., 2017).

Social targets' smiling and laughing expressions were evoked by comical audio clips. Their gaze was directed toward the camera. Auditory stimuli were chosen based on social targets' preferences and were retrieved from the internet (e.g., http://www.youtube.com). After each expression, social targets were asked to provide subjective evaluations of the enjoyment felt while making the expression. First, they were asked to report if they were feeling enjoyment or not. If they reported having felt enjoyment, they were asked to rate it using a 9-point Likert scale $(1=$ minimal enjoyment; 9 = maximal enjoyment). Moreover, they were asked to evaluate their subjective confidence in their enjoyment rating using a categorical response (I am 100\% sure of my judgment/I am not 100\% sure of my judgment) (Paracampo et al., 2017). Stimuli for which social targets reported no enjoyment or uncertainty in the judgment were excluded from the present study. In a separate session, social targets performed the EA task on a set of $\sim 100$ movies depicting themselves while smiling/laughing. Social targets were asked to observe each clip and rate the enjoyment they experienced while smiling/laughing, using the same 9-point Likert scale used in the recording session. The EA task was repeated three times. 


\section{A}

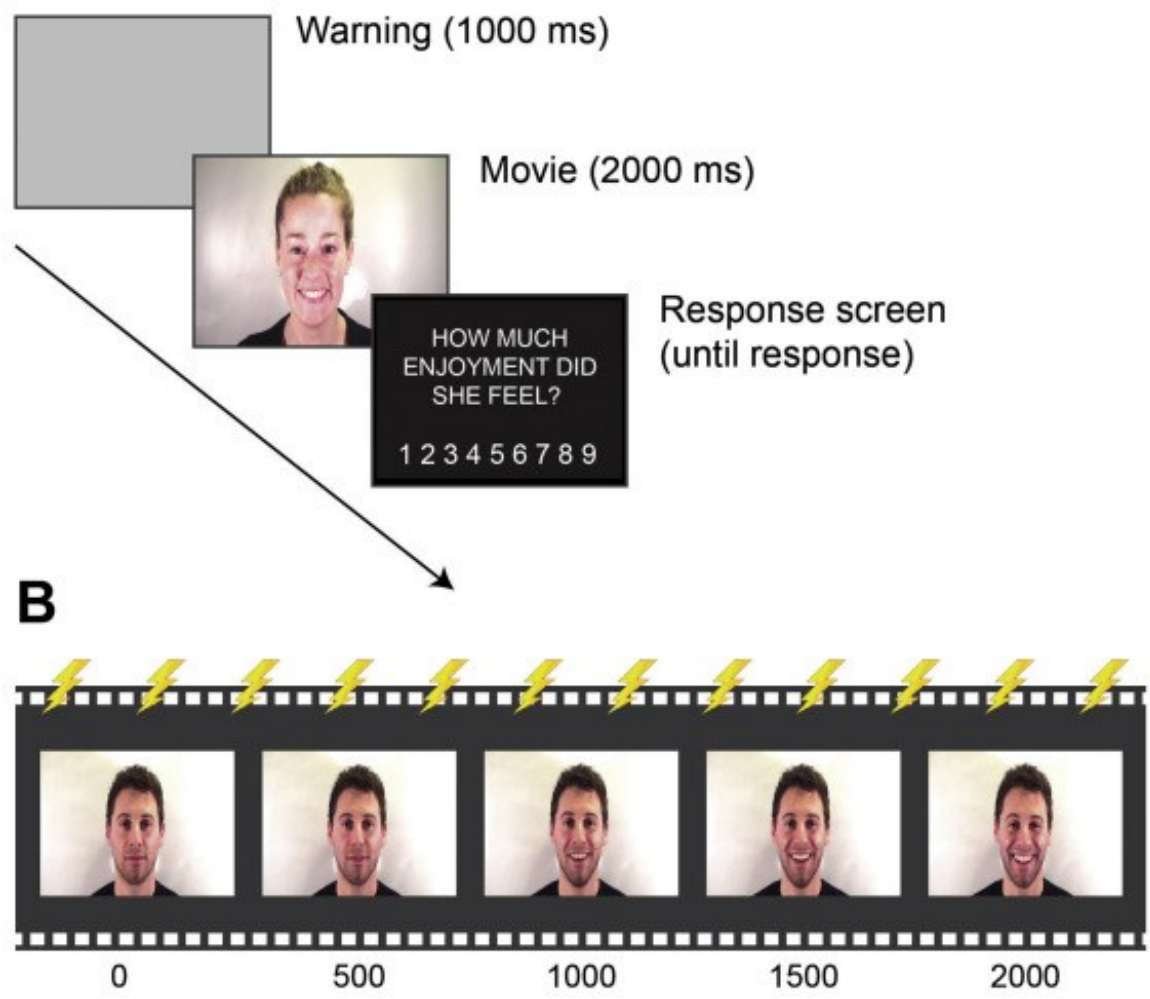

Fig. 1. Trial example for the EA task (A). Participants were asked to rate the enjoyment felt by the actor on a 9-point Likert-scale. A continuous 6-Hz train of 12 pulses of rTMS was applied at the onset of the movies (B).

These behavioral data were used to preselect 300 movies (50 for each social target) in order to sample different levels of enjoyment, and guarantee stimulus variability while removing smiles/laughter associated with inconsistent judgments. For each social target, the $\sim 100$ movies were grouped into 3 enjoyment categories based on the mean enjoyment score across the 4 evaluations [i.e., low $(\leq 3.25)$, mid (3.5-6.25), and high $(\geq 6.5)$ enjoyment scores]. For each social target and enjoyment category, we selected 18 movies associated with the most consistent rating (i.e., the lowest SD across the 4 evaluations). Movies with gross head movements or video artifacts were also excluded, reaching a total of 300 movies (50 movies for each of the six social targets; 100 movies for each low/mid/high enjoyment category).

The final set of 90 movies for the rTMS experiments was selected based on the results of a pilot study conducted on 24 participants. Participants in the pilot study performed the EA task on the 300 preselected movies: they were asked to watch each movie and judge the enjoyment they believed social targets had felt during each smiling/laughing expression using a 9-point Likert scale ( 1 = minimal enjoyment; 9 = maximal enjoyment). Participants' ratings and social targets' ratings were then correlated to provide a measure of EA (Zaki et al., 2008, Zaki et al., 2009a). For each 
participant and social target, Pearson's $r$ coefficients were computed across the social target's 50 movies. We selected 15 movies for each social target (90 movies in total) to obtain moderate and comparable EA scores across the six social targets (mean EA: $r=0.57$ ). To guarantee stimulus variability, we also ensured that the final sample comprised 30 movies from each of the low/mid/high enjoyment categories for each social target, and covered the entire range of judgments (range: 1-9; mean: 5; SD: 2.3).

The final selection of 90 movies was further validated by two Facial Action Coding System (FACS) judges. According to the FACS, two facial action units are consistently observed in smiles of enjoyment (Ekman and Friesen, 1982, Frank and Ekman, 1993, Hess and Kleck, 1994, Ambadar et al., 2009, McLellan et al., 2010). These are coded as Action Unit 6 (AU6) and Action Unit 12 (AU12), and are produced by activation of the orbicularis oculi (i.e., the muscle that makes crow's feet at the outer corner of the eye) and the zygomaticus major (i.e., the muscle that extends the mouth and acts as a lip corner puller), respectively. The two judges were blind to the social targets' ratings and were asked: i) to verify whether all smiles contained activations of both AU6 and AU12; ii) to rate the intensity of each $\mathrm{AU}$ on an ordinal scale from $\mathrm{A}$ (traces of $\mathrm{AU}$ activation) to $\mathrm{E}$ (maximum AU activation) (McLellan, 2010). The two FACS judges confirmed that all our movies displayed a neutral face rapidly turning into a weak or strong smile involving the activation of both AU6 and AU12. Inter-rater reliability between the intensity ratings of the two judges was high (Cohen's kappa $=0.86, P<0.001$ ).

Moreover, we tested whether the FACS coding (the average of the two judges' ratings) would map onto the actors' subjective rating of enjoyment. To this aim, we investigated the correlation between the activation intensity of each AU6/AU12 and the social target's enjoyment ratings for each smile. Since AU intensity ratings are on an ordinal scale, nonparametric Spearman's Rho coefficients $\left(r_{s}\right)$ were computed. The results showed that the social targets' ratings of felt enjoyment were correlated with the intensity of both AU6 $\left(r_{s}=0.72, P<0.001\right)$ and AU12 $\left(r_{s}=\right.$ $0.45, P<0.001)$.

\subsection{Procedure}

Experiments were programmed using Matlab to control video clip sequence, rTMS triggering, and behavioral performance recording. Participants were initially tested in an electrophysiological and neuronavigation session in which the intensity of rTMS and coil position were determined (see below). Then, they were presented with task instructions, and performed an EA training task on a subset of ten stimuli not included in the main session.

In the rTMS experiments, participants performed the EA task during three rTMS conditions: a sham rTMS condition serving as a baseline for EA performance and two active rTMS conditions 
that varied between the two experiments. In each rTMS condition, participants were presented with the entire set of 90 movies ( 270 trials in total) that were divided in 3 blocks of 30 EA trials each. The 9 blocks were presented in a counterbalanced order across participants.

Each trial started with a gray screen $(1000 \mathrm{~ms})$ followed by the movie $(2000 \mathrm{~ms})$. After the movie, a response prompt appeared, and it remained on the screen until the participant's response (Fig. 1A). Participants provided their response by pressing one of 9 keys on the keyboard. They were asked to answer as quickly and accurately as possible using their right hand. After the keypress, the response screen was replaced by a black screen (inter-trial interval: 5000-6000 ms). On each trial, a time-locked single train of $6 \mathrm{~Hz}$ rTMS (12 pulses) was administered using a figure-of-eight coil (diameter: $70 \mathrm{~mm}$ ) connected to a Magstim Rapid2 stimulator (Magstim).

In each rTMS experiment, the coil was placed tangentially over two target brain regions (V1 and STS in Experiment 1; IFG and TPJ in Experiment 2) or tilted at $90^{\circ}$ over the vertex (sham rTMS), to provide some scalp sensations and rTMS sounds similar to active rTMS but without inducing a current in the brain. The rTMS train was administered at the onset of each movie and covered its entire duration ( $2 \mathrm{~s}$; see Fig. 1B). Stimulation intensity corresponded to $90 \%$ of the resting motor threshold (rMT; see below) and did not differ between the two groups (Experiment 1: rTMS intensity \pm SD: $49 \% \pm 4$ of maximum stimulator output; Experiment 2: $46 \% \pm 4$; comparison: $t_{30}=$ $-1.89, P=0.07)$.

At the end of the rTMS session, participants were asked to evaluate the discomfort caused by active rTMS on a 5-point Likert scale with 1 indicating "not unpleasant at all" and 5 corresponding to "extremely unpleasant". Moreover, they were asked to report whether rTMS induced phosphenes. None of the participants reported phosphenes after rTMS over V1 or other brain regions.

Finally, participants were asked to complete two subscales of Davis' Interpersonal Reactivity Index (IRI) (Davis, 1983): the Perspective Taking (PT) and Empathic Concern (EC) subscales. The PT subscale assesses cognitive components of empathy, i.e., the tendency to spontaneously imagine and assume the cognitive perspective of another person. The EC subscale assesses the tendency to feel sympathy and compassion for others in need, and it is considered to assess emotional empathy (Davis, 1996). In this study, we assessed PT and EC to ensure that participants in Experiment 1 and Experiment 2 did not differ in dispositional empathy, in addition to baseline EA performance. An exploration of the influence that dispositional empathy exerts over EA performance and how rTMS affects that influence is not the focus of the present study, and will be presented in a separate publication (Paracampo and Avenanti, in prep).

\subsection{Electrophysiological and neuronavigation sessions}


To set rTMS intensity, the rMT was estimated for all participants in a preliminary phase of the experiment using standard procedures (Rossi et al., 2009). Motor-evoked potentials (MEPs) induced by stimulation of the right motor cortex were recorded from the left first dorsal interosseous (FDI) by means of a Biopac MP-35. Electromyography (EMG) signals were bandpass filtered $(30-500 \mathrm{~Hz})$ and digitized at a sampling rate of $5 \mathrm{kHz}$. Pairs of $\mathrm{Ag}-\mathrm{AgCl}$ surface electrodes were placed in a belly-tendon montage with a ground electrode on the wrist. The intersection of the coil was placed tangentially to the scalp with the handle pointing backward and laterally at a $45^{\circ}$ angle away from the midline. The optimal scalp position for inducing MEPs from the left FDI was first localized, and the rMT was determined from that position. The rMT was defined as the minimal intensity of stimulator output that produced MEPs with an amplitude of at least $50 \mu \mathrm{V}$ in the FDI with $50 \%$ probability (Rossini et al., 2015).

Each brain area was individually targeted using image-guided neuronavigation. The coil position was identified on each participant's scalp using the SofTaxic Navigator System (Electro Medical Systems). Skull landmarks (nasion, inion, and 2 preauricular points) and $\sim 80$ points providing a uniform representation of the scalp were digitized by means of a Polaris Vicra digitizer (Northern Digital), as in our previous research (Avenanti et al., 2007, Avenanti et al., 2012, Tidoni et al., 2013, Jacquet and Avenanti, 2015, Paracampo et al., 2017). An individual estimated magnetic resonance image (MRI) was obtained for each subject through a 3D warping procedure that fits a high-resolution MRI template with the participant's scalp model and craniometric points. This procedure has been proven to ensure a global localization accuracy of roughly $5 \mathrm{~mm}$, a level of precision close to that obtained using individual MRIs (Carducci and Brusco, 2012). Stimulation sites were identified on the basis of previous fMRI studies, using the SofTaxic Navigator (STS, IFG, TPJ) or established anatomical methods (V1). For STS, IFG and TPJ, Talairach coordinates of target regions and their corresponding scalp projections were automatically estimated by the SofTaxic Navigator from the MRI-constructed stereotaxic template. When necessary, coordinates in Talairach space were obtained by converting MNI coordinates reported in previous studies using GingerALE 2.3.1. The V1 site was identified at $2 \mathrm{~cm}$ above the inion. Its location was consistent with previous TMS studies using anatomical landmarks or functional procedures (i.e., phosphene induction) (Silvanto et al., 2005, Koivisto et al., 2010, Romei et al., 2016). This site was selected as a control site in other studies addressing neural bases of social perception (e.g., Urgesi et al., 2004, Urgesi et al., 2007; Pobric and Hamilton, 2006). The right STS site was localized in its posterior sector over the Talairach coordinates: $\mathrm{x}=$ $48, y=-49, z=4$, derived by converting the MNI coordinates identified by a meta-analysis of 100 neuroimaging studies contrasting emotional face $>$ neutral face (Sabatinelli et al., 2011). The right IFG scalp site was localized based on the following Talairach coordinates: $\mathrm{x}=47, \mathrm{y}=8, \mathrm{z}=$ 28 , which were identified on the basis of previous fMRI meta-analyses exploring activations 
associated with the execution and/or observation of facial movements and emotional expressions (Caspers et al., 2010, Grosbras et al., 2012, Molenberghs et al., 2012). This site corresponds to the posterior sector of the IFG (at the border with the ventral premotor cortex), which plays a role in sensorimotor simulation (Avenanti et al., 2007, Avenanti et al., 2013a). The right TPJ site was localized based on the following Talairach coordinates: $x=51, y=-54, z=21$, which were identified on the basis of neuroimaging studies exploring areas related to mentalizing (van Overwalle and Baetens, 2009, Mar, 2011, Bzdok et al., 2012). The chosen coordinates are also in line with brain stimulation studies targeting STS and IFG to affect action and emotion perception (e.g., Urgesi et al., 2007; Cattaneo et al., 2010; Candidi et al., 2011; Pitcher, 2014; Paracampo et al., 2017; Avenanti et al., 2017) and TPJ to affect mental state attribution and emotion recognition (Costa et al., 2008, Young et al., 2010, Santiesteban et al., 2012, Donaldson et al., 2015; Gamond and Cattaneo, 2016).

Locations of scalp regions identified by neuronavigation (STS, IFG, TPJ) or anatomical methods (V1) were marked with a pen on each participant's head and used to place the rTMS coil. Then, individual Talairach coordinates corresponding to the projection of the targeted scalp sites onto the surface of the MRI-constructed stereotaxic template were automatically estimated through the neuronavigation system. These estimated coordinates indicate the most superficial cortical site where rTMS effects are expected to be maximal. Brain surface coordinates were converted to MNI space (using GingerALE 2.3.1) for visualization with MRIcron software (MRIcron/NPM/dcm2nii). Fig. 2 illustrates the estimated group mean MNI surface coordinates.

\subsection{Data analysis}

Behavioral data were processed offline. For each participant, an EA score was calculated as the correlation between the participant's ratings and the social targets' ratings (Zaki et al., 2009a, Zaki et al., 2009b, Harvey et al., 2013). EA scores were computed separately for each rTMS condition (sham, V1 and STS for Experiment 1; sham, IFG and TPJ for Experiment 2). To obtain a summary measure of performance speed that is relatively uninfluenced by spuriously fast or slow responses (Stuart and Ord, 1987), individual median response times (RTs) were computed for each rTMS condition after removing outlier values deviating more than 2 SD from the median value (fewer than 5\%). As a primary outcome measure, a global index of EA task efficiency, i.e., the inverse efficiency (IE) score, was computed by merging indices of performance speed and accuracy into a single measure. IE scores are commonly calculated by dividing the mean RT by the proportion of correct responses (Townsend and Ashby, 1983, Pernigo et al., 2012, Jacquet and Avenanti, 2015, Penton et al., 2017). For each rTMS condition, we computed a modified version of the IE score as the ratio between our measures of performance speed (median RT) and accuracy (EA 
score), to discount possible criterion shifts or speed-accuracy trade-offs induced by active rTMS. The higher the IE score, the poorer the performance. In other words, higher EA efficiency is associated with lower IE scores. Mean enjoyment ratings were also computed for each rTMS condition.

A series of independent samples $t$-tests were preliminarily performed between Experiment 1 and 2 to ensure that participants in the two experiments showed similar dispositional empathy (IRI PT and EC scores) and comparable EA task performance (IE scores and raw mean enjoyment ratings) in the baseline condition (i.e., in the sham rTMS condition).

Experiment 1

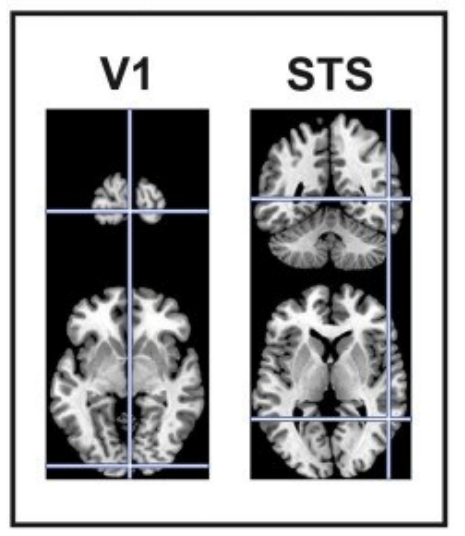

\section{Experiment 2}

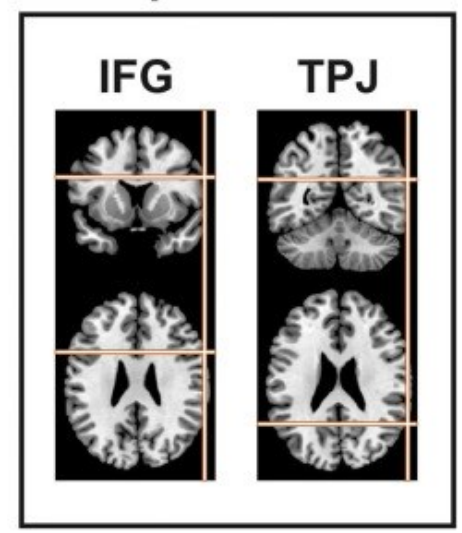

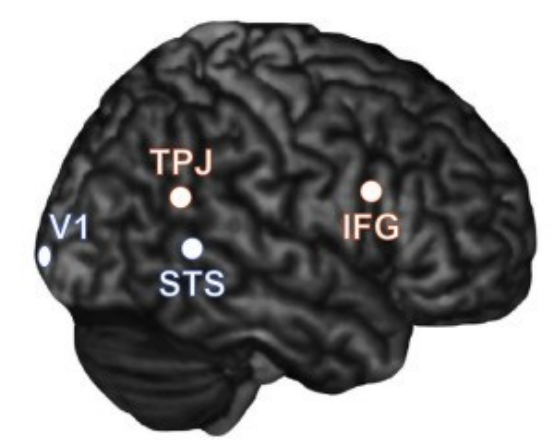

Fig. 2. Schematic representation of the brain stimulation sites reconstructed on a standard template using MRIcron (MRIcron/NPM/dcm2nii). Talairach coordinates corresponding to the projection of the V1, STS, IFG, and TPJ scalp sites onto the brain surface were individually estimated through the neuronavigation system. Group mean brain surface coordinates \pm SEM for the V1 site were: $x=0.3 \pm$ $0.9 ; y=-91.1 \pm 2.1 ; z=-6.7 \pm 3.2$. Coordinates for the STS were: $x=54.6 \pm 1.2 ; y=-51.0 \pm 0.4 ; z=$ $3.7 \pm 0.2$. Coordinates for the IFG were: $x=53.2 \pm 0.4 ; y=7.8 \pm 0.1 ; z=29.1 \pm 0.1$. Coordinates for the TPJ were: $x=54.9 \pm 0.2 ; y=-54.3 \pm 0.2 ; z=21.7 \pm 0.2$. Talairach coordinates were then converted into MNI space (using GingerALE 2.3.1) for visualization with MRIcron software. 
For each experiment, a separate one-way ANOVA was performed on the primary outcome measure (IE scores) with rTMS condition as a 3-level within-subjects factor (V1, STS, and sham in Experiment 1; IFG, TPJ, and sham in Experiment 2). Post-hoc analyses were performed using the Newman-Keuls test to correct for multiple comparisons. Further one-way ANOVAs and posthoc analyses (when necessary) were performed on secondary outcome measures (raw enjoyment ratings, EA scores and RTs; see Table S1).

To ensure that active rTMS did not cause different levels of discomfort over the different areas stimulated in Experiment 1 and 2, a series of independent and paired samples $t$-tests were performed. Moreover, in both experiments, we checked whether interference effects of rTMS (the difference in IE scores between active and sham rTMS) were correlated with discomfort ratings. Lastly, we tested whether individual differences in EA moderated the effects of rTMS. To this aim, we first explored whether baseline EA efficiency (i.e., during sham rTMS) predicted changes in efficiency following active rTMS. To do so, for each target region, a regression analysis was performed with baseline IE scores as a predictor and the interference effect of rTMS (the difference in IE scores between active and sham rTMS) as the dependent variable. Ratings of discomfort were also added to the model as a predictor of no interest. Cohen's effect size $\left(f^{2}\right)$ of regression coefficients was calculated using the formula $f^{2}=R^{2} /\left(1-R^{2}\right)$ (Cohen, 1992). Additionally, we performed a median split on IE scores in the sham rTMS condition to create two groups of lowand high-performers at baseline. Then, a mixed factors 2-way ANOVA was performed with baseline performance as a between-subjects factor (2 levels: low-performers, high-performers) and rTMS condition as a within-subjects factor (3 levels: V1, STS, and sham for Experiment 1; IFG, TPJ, and sham for Experiment 2).

In all the ANOVAs, partial eta squared $\left(\eta_{p}^{2}\right)$ was computed as a measure of effect size for significant main effects and interactions, whereas Cohen's $d$ indices were computed for $t$-tests and post-hoc comparisons. For within-subjects comparisons, $d$ indices were computed following the recommendation of Dunlap et al. (1996), using the formula $d=t[2(1-r) / \mathrm{n}]^{1 / 2}$, where $t$ is the statistic for paired samples and $r$ is the correlation across pairs of means (Dunlap et al., 1996, Morris and DeShon, 2002).

All $t$-tests, ANOVAs, correlations and regressions were conducted using the software STATISTICA v. 12 with the significance level set at 0.05 . These null hypothesis-testing analyses were complemented by their Bayesian implementations using JASP v 0.8.4 (Jasp Team, 2017). With Bayesian hypothesis testing, we could directly evaluate the relative strength of evidence for the null and alternative hypotheses, providing quantification of the degree to which the data support either hypothesis (Dienes, 2011, Wagenmakers et al., 2017). We used default priors in JASP for Bayesian $t$-tests (zero-centered Cauchy prior width, $r=0.707$ ), ANOVAs ( $r$ scale fixed effects $=0.5 ; r$ scale random effects $=1$ ), correlations (stretched beta prior width, $r=1$ ) and regression 
analyses $(r$ scale covariates $=0.354)$. Following the current standards, we report subscripts on Bayes Factors to refer to the models compared. Accordingly, the Bayes Factor for the alternative relative to the null hypothesis is denoted $\mathrm{BF}_{10}$, while the Bayes Factor for the null relative to the alternative hypothesis is denoted $\mathrm{BF}_{01}$. We interpreted and labelled the sizes of $\mathrm{BFs}$ according to the recommendations of Raftery (1995) as referred to by Jarosz and Wiley (2014).

\section{Results}

\subsection{Preliminary analyses}

Participants in the two experiments showed similar EA task performance in the baseline condition (sham rTMS). Table 1 shows no difference between groups for our primary outcome measure of EA task efficiency, i.e., IE score $(P=0.97$, Cohen's $d=0.01)$. Similarly, no between-group differences emerged for raw mean enjoyment ratings, nor for the Empathic Concern and Perspective Taking subscales of Davis' IRI questionnaire (all $P>0.70$, all Cohen's $d<0.14$ ). For all variables, Bayesian hypothesis testing provided positive evidence in favor of the null hypothesis of no difference between groups (all $\mathrm{BF}_{01}$ > 3.7). Thus, random allocation of participants to Experiment 1 and 2 created two groups with similar baseline EA and dispositional empathy. This implies that any differential effects induced by active rTMS in the two experiments cannot be due to systematic between-group differences in empathy at baseline.

\begin{tabular}{|c|c|c|c|c|c|c|}
\hline & $\begin{array}{c}\text { Experiment } 1 \\
\text { sham rTMS }\end{array}$ & $\begin{array}{c}\text { Experiment } 2 \\
\text { sham rTMS }\end{array}$ & t-test & $\mathbf{P}$ & Cohen's d & $\mathrm{BF}_{01}$ \\
\hline $\begin{array}{l}\text { Inverse efficiency } \\
\text { (IE) scores [RTs/EA] }\end{array}$ & $2.50 \pm 0.71$ & $2.49 \pm 0.57$ & $t_{30}=0.04$ & $p=0.97$ & $d=0.01$ & $B F_{0 I}=3.9$ \\
\hline Enjoyment ratings & $5.03 \pm 0.94$ & $5.12 \pm 0.95$ & $t_{30}=0.33$ & $p=0.75$ & $d=0.12$ & $B F_{01}=3.7$ \\
\hline $\begin{array}{r}\text { Empathic concern } \\
(\text { EC) }\end{array}$ & $17.2 \pm 3.4$ & $17.4 \pm 4.9$ & $t_{30}=0.38$ & $p=0.70$ & $d=0.06$ & $B F_{01}=3.9$ \\
\hline $\begin{array}{r}\text { Perspective taking } \\
\text { (PT) }\end{array}$ & $20.1 \pm 3.6$ & $20.7 \pm 4.5$ & $t_{30}=0.17$ & $p=0.87$ & $d=0.14$ & $B F_{01}=3.7$ \\
\hline
\end{tabular}

Table 1. Mean $\pm S D$ values of inverse efficiency (IE) scores, enjoyment ratings, and interpersonal reactivity index's empathic concern (EC) and perspective tasking (PT) subscale. For each measure independent t-tests, effect sizes and Bayesian independent t-tests are reported. 


\subsection{Experiment 1: rTMS of STS, but not of V1, disrupts the efficiency of EA task}

performance

The ANOVA conducted on the global score of EA task performance (IE scores) was significant $\left(F_{2,30}=7.43, P=0.002 ; \eta_{p}{ }^{2}=0.33\right.$; Fig. $\left.3 \mathrm{~A}\right)$ and the Bayesian ANOVA showed positive evidence favoring the alternative hypothesis $\left(\mathrm{BF}_{10}=14.5\right)$. Post-hoc analysis showed that IE scores were increased (indexing a reduction in performance efficiency) following active rTMS over STS (mean IE scores \pm SD: $3.08 \pm 1.27)$ relative to the sham rTMS $(2.50 \pm 0.71 ; P=0.003 ;$ Cohen's $d=0.80)$ and active rTMS over V1 $(2.63 \pm 1.14 ; P=0.008$; Cohen's $d=0.92)$, which in turn did not differ from one another $(P=0.42$; Cohen's $d=0.20)$. These findings indicate that active stimulation of STS, but not of V1, reduced the ability to efficiently infer the enjoyment felt by social targets from their smiles/laughter.

A

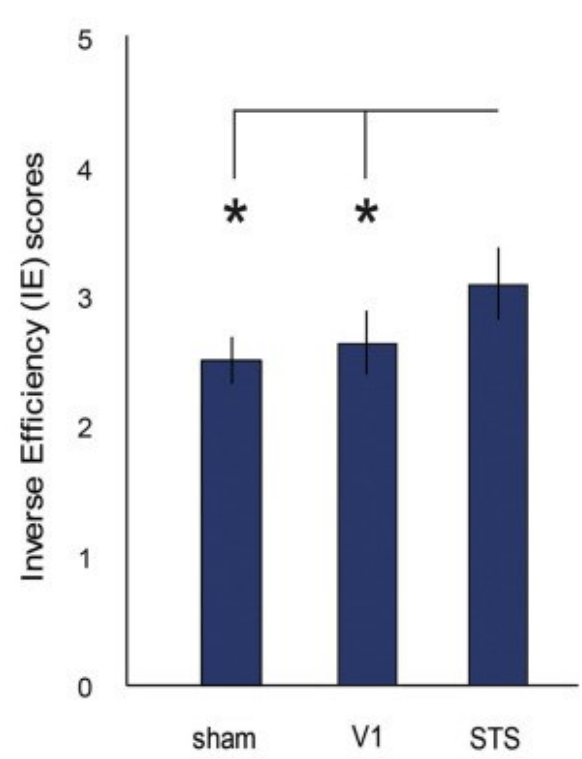

B

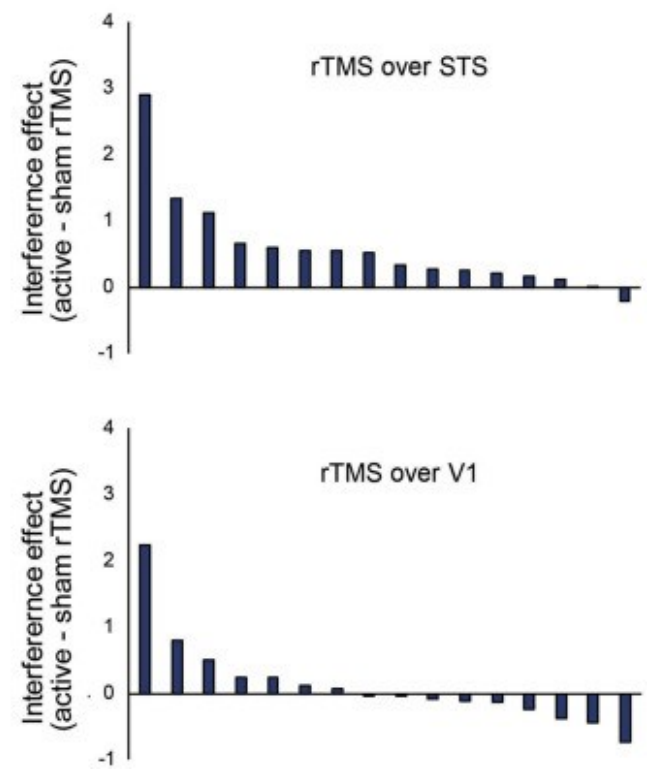

Fig. 3. (A) Inverse efficiency (IE) scores for the three rTMS conditions of Experiment 1. Active rTMS of STS increased IE scores (i.e., disrupted task efficiency) compared to baseline (sham rTMS) and rTMS of V1. Asterisks indicate significant post-hoc comparisons $(\mathrm{P}<0.05)$. Error bars denote

SEM. (B) Interferential effect of $r T M S$ (difference in IE scores between active and sham rTMS) across participants in Experiment 1. Effects of STS (top) and V1 (bottom) stimulation. Positive and negative values indicate reductions and increases in performance, respectively.

Fig. 3B shows the interference effect of rTMS (difference in IE scores between active and sham rTMS) across participants. Stimulation of STS tended to consistently increase IE scores at the 
group level, with 15 out of 16 participants showing positive values (less efficient EA task performance), although the magnitude of the interference was variable across participants. Stimulation of V1 tended to induce a more symmetric distribution of interference values centered at zero, with 7 and 9 participants showing increases and decreases in IE scores, respectively, and no net change in performance.

To understand whether the reduction of EA task efficiency in Experiment 1 was due to changes in accuracy, speed or both, follow up ANOVAs were conducted on EA scores and RTs separately. Table S1 shows that, in Experiment 1, the increase in IE scores due to STS stimulation was mainly due to a reduction in EA scores. RTs showed a non-significant increase following STS stimulation, thus ruling out any speed-accuracy trade off.

The ANOVA on participants' raw enjoyment ratings showed no consistent effect of rTMS $\left(F_{2,30}=1.70, P=0.20 ; \eta_{p}{ }^{2}=0.10 ; \mathrm{BF}_{01}=2.0\right.$; see Table 2$)$, thus suggesting that rTMS over STS did not systematically increase or decrease enjoyment ratings but, rather, hindered their accuracy.

\begin{tabular}{|r|c|c|c|c|c|c|}
\cline { 2 - 7 } \multicolumn{1}{c|}{} & \multicolumn{3}{c|}{ Experiment 1 } & \multicolumn{3}{c|}{ Experiment 2 } \\
\cline { 2 - 7 } & sham & V1 & STS & sham & IFG & TPJ \\
\hline Enjoyment \\
ratings
\end{tabular}

Table 2. Mean $\pm S D$ values of enjoyment ratings in the rTMS conditions of Experiment 1 and 2.

\subsection{Experiment 2: rTMS of IFG and TPJ disrupts the efficiency of EA task performance}

The ANOVA conducted on IE scores was significant $\left(F_{2,30}=4.79, P=0.016 ; \eta_{p}{ }^{2}=0.24\right.$; Fig. 4A) and the Bayesian ANOVA showed positive evidence favoring the alternative hypothesis $\left(\mathrm{BF}_{10}=4.5\right)$. Relative to sham rTMS $(2.49 \pm 0.57)$, IE scores were increased (indexing a reduction in task performance) during active rTMS over IFG (3.05 $\pm 1.01 ; P=0.036$; Cohen's $d=0.83)$ and TPJ ( $3.25 \pm 0.97 ; P=0.036$; Cohen's $d=0.84)$; no difference in IE scores was found between the two active rTMS conditions ( $P=0.44$; Cohen's $d=0.15$ ). These findings indicate that active stimulation of both IFG and TPJ reduced the ability to efficiently infer the enjoyment felt by social targets from their smiles/laughter. 

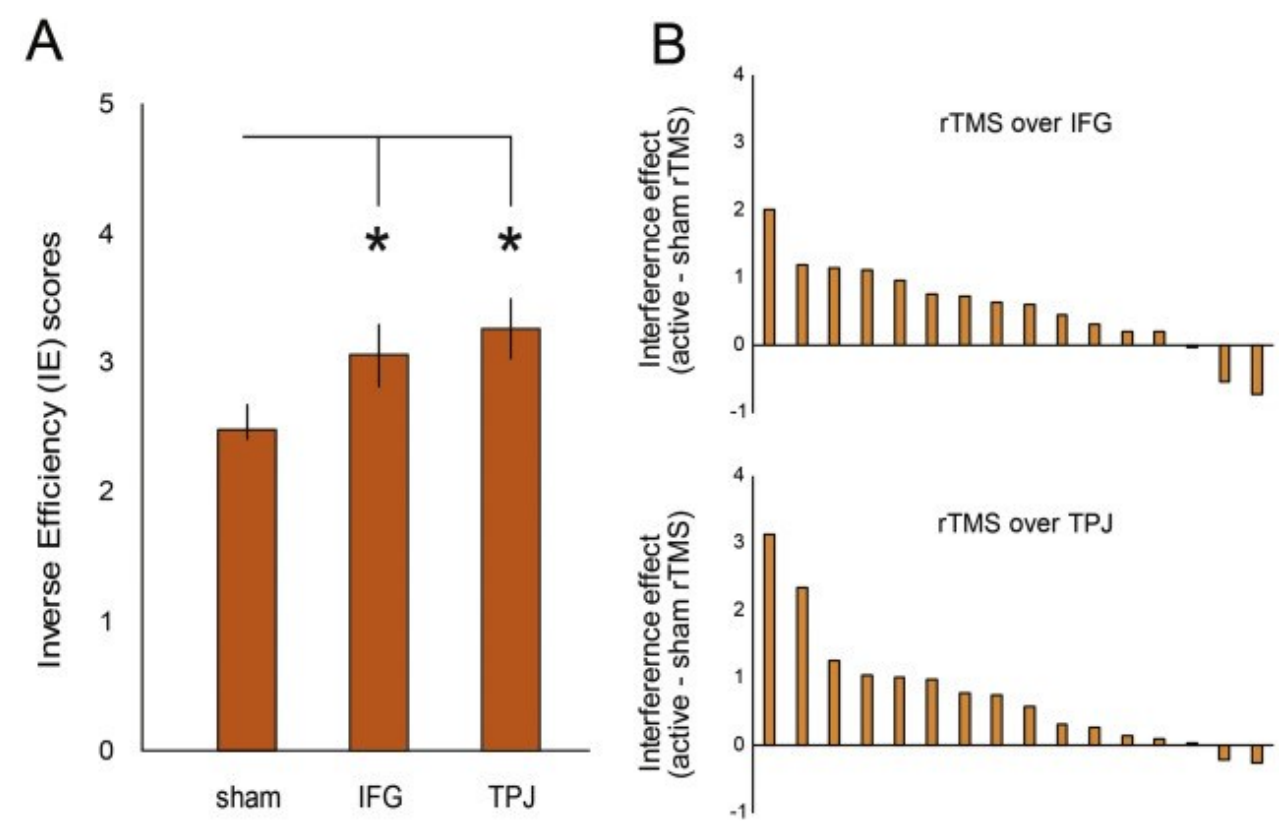

Fig. 4. (A) Inverse efficiency (IE) scores for the three rTMS conditions of Experiment 2. Active rTMS of IFG and TPJ increased IE scores (disrupted task efficiency) compared to baseline (sham rTMS). Asterisks indicate significant post-hoc comparisons $(\mathrm{P}<0.05)$. Error bars denote SEM. (B) Interferential effects of $r T M S$ (difference in IE scores between active and sham rTMS) across participants in Experiment 2. Effects of IFG (top) and TPJ (bottom) stimulation. Positive and negative values indicate reductions and increases in performance, respectively.

Fig. 4B shows the interference effect of rTMS (difference in IE scores between active and sham rTMS) across participants. Stimulation of both IFG and TPJ tended to consistently increase IE scores at the group level, with 14 out of 16 participants showing positive values (less efficient EA task performance), although the magnitude of the interference was variable across participants. Follow-up analyses conducted on EA scores and RTs separately showed that, in Experiment 2, stimulation of IFG significantly reduced EA scores and marginally increased RTs (Table S1); moreover, stimulation of TPJ significantly reduced EA scores and increased RTs (Table S1). The ANOVA on participants' raw enjoyment ratings showed no consistent effect of rTMS $\left(F_{2,30}=2.04, P=0.15 ; \eta_{p}{ }^{2}=0.12 ; \mathrm{BF}_{01}=1.7\right.$; Table 2). Thus, as in Experiment 1 , rTMS did not systematically increase or decrease enjoyment ratings in Experiment 2 but, rather, reduced their accuracy. The reduction in accuracy following active rTMS over IFG and TPJ was accompanied by a reduction in response speed, thus ruling out that disruption in EA task performance was due to any speed-accuracy trade-offs.

\subsection{Discomfort ratings}


We checked whether the unpleasantness of the stimulation could explain our results. Thus, we analyzed discomfort ratings reported by participants after active rTMS in the two experiments (Table 3). A series of $t$-tests were performed, both within and between experiments. No significant differences emerged when comparing active TMS conditions within experiments, neither in Experiment $1\left(t_{15}=0.22 ; P=0.83\right.$; Cohen's $\left.d=0.06\right)$ nor in Experiment $2\left(t_{15}=1.17 ; P=\right.$ 0.26 ; Cohen's $d=0.29)$. Moreover, no differences emerged when comparing active TMS conditions between experiments (all $t_{30}<0.51$; all $P>0.61$; all Cohen's $d<0.18$ ). Bayesian $t$-tests showed weak-to-positive evidence in favor of the null hypothesis of no difference between discomfort ratings across the different rTMS conditions $\left(2.2<\mathrm{B}_{01}<3.8\right)$.

\begin{tabular}{|r|c|c|c|c|}
\cline { 2 - 5 } \multicolumn{1}{c|}{} & \multicolumn{2}{c|}{ Experiment 1 } & \multicolumn{2}{c|}{ Experiment 2 } \\
\cline { 2 - 5 } & V1 & STS & IFG & $2.6 \pm 1.1$ \\
\hline Discomfort ratings & $2.8 \pm 1.0$ & $2.8 \pm 0.9$ & $2.9 \pm 0.8$ & $r=0.12$ \\
\hline Discomfort and & $r=0.31$, & $r=0.19$, & $r=0.03$, & $P=0.67$ \\
\hline
\end{tabular}

Table 3. Mean $\pm S D$ values of discomfort ratings for the active TMS conditions in Experiment 1 (STS and V1) and 2 (IFG and TPJ) and correlation between discomfort ratings and interferential effect of $r$ TMS (difference in IE scores between active and sham rTMS).

Lastly, we checked whether discomfort ratings predicted the interferential effect of rTMS (difference in IE scores between active and sham rTMS). As shown in Table 3, no significant correlation was found between discomfort ratings and changes in IE scores following stimulation of STS, IFG or TPJ $(0.03<r<0.19$, all $P>0.49)$ and data showed weak-to-positive evidence in favor of the null hypothesis of no correlation $\left(2.6<\mathrm{B}_{01}<3.2\right)$.

Thus, the effects of STS, IFG and TPJ stimulation on EA task performance, and the absence of effects with V1 stimulation, were not due to any unspecific difference in the discomfort caused by active rTMS, but, rather, reflected the causal involvement of STS, IFG and TPJ in EA task performance.

\subsection{Individual differences in baseline $\mathbf{E A}$ task efficiency reveal differential causal involvements of visual, sensorimotor and cognitive mechanisms}

To delineate the relationship between baseline EA efficiency and performance change following rTMS, a linear regression was run. Baseline EA task performance (indexed by IE scores) was 
entered as a predictor and the interferential effect of rTMS (difference in IE scores between sham rTMS and active rTMS) was entered as the dependent variable.

In Experiment 1, baseline performance predicted the performance change following stimulation of STS $\left(\beta=0.55 ; t_{14}=2.42, P=0.03\right)$ and the regression model showed a large effect size (Adjusted $R^{2}=0.24, F_{1,14}=5.84, P=0.03 ; f^{2}=0.42$; no outliers with standard residual $>2.5 \sigma$ in the dataset), with low-performers showing the greatest impairment in performance following rTMS over STS. Baseline EA task performance also remained a significant predictor when controlling for the discomfort caused by STS-rTMS ( $\left.\beta=0.67, t_{13}=3.07, P=0.009\right)$. This pattern was not found following rTMS of V1 (Adjusted $R^{2}=0.06, F_{1,14}=1.93, P=0.19 ; f^{2}=0.14$; baseline performance alone: $\beta=0.35, t_{14}=1.39, P=0.19$; baseline performance plus discomfort: $\beta=0.26, t_{13}=1.00, P=$ 0.33 ). Bayesian regression showed positive evidence in favor of the hypothesis that baseline performance predicts the performance change following STS stimulation $\left(\mathrm{BF}_{10}=6.4\right)$ relative to the null model (including the effect of discomfort). In the case of V1 stimulation, the data showed weak evidence in favor of the null hypothesis $\left(\mathrm{BF}_{01}=1.2\right)$.

These results were confirmed in a further analysis. We conducted a median split on IE scores in the sham rTMS condition of Experiment 1 to create two groups of low- and high-performers at baseline. Then, a Baseline (low-performers, high-performers) x rTMS condition (sham, V1, STS) mixed factors ANOVA was conducted on IE scores (Fig. 5A).

\section{Experiment 1}

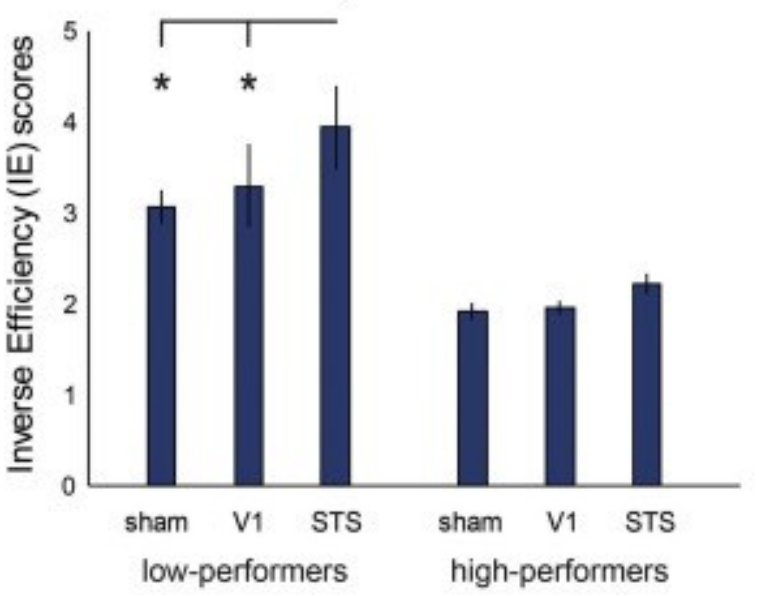

Experiment 2

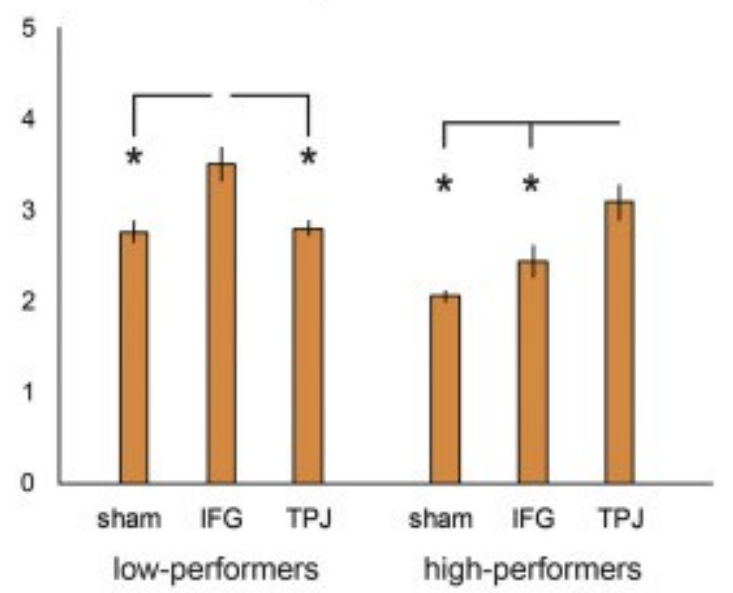

Fig. 5. (A) Inverse efficiency (IE) scores for low-performers (left) and high-performers (right) in the three rTMS conditions of Experiment 1. Only in low-performers, active rTMS of STS disrupted task efficiency compared to baseline (sham rTMS) and rTMS of V1. Asterisks indicate significant planned comparisons $(\mathrm{P}<0.05)$. Error bars denote SEM. (B) IE scores for low-performers (left) and highperformers (right) in the three rTMS conditions of Experiment 2. In low-performers, active rTMS of IFG disrupted task efficiency compared to sham rTMS and rTMS of TPJ, whereas in high-performers, active 
rTMS of TPJ disrupted task efficiency compared to sham rTMS and rTMS of IFG. Asterisks indicate significant post-hoc comparisons $(\mathrm{P}<0.05)$. Error bars denote SEM.

The ANOVA showed a main effect of Baseline $\left(F_{1,14}=15.25, P=0.002 ; \eta_{p}^{2}=0.52\right)$, with highperformers showing lower IE scores than low-performers, and a main effect of rTMS condition $\left(F_{2,28}=7.81, P=0.002 ; \eta_{p}{ }^{2}=0.36\right)$, confirming the increase in IE scores following STS stimulation. The Baseline $\mathrm{x}$ rTMS condition interaction did not reach statistical significance $\left(F_{2,28}=1.78, P=0.19 ; \eta_{p}{ }^{2}=0.11\right)$. A Bayesian ANOVA with the factors Baseline and rTMS condition showed that all the alternative models received positive/strong support in comparison to the null model (all $\mathrm{BF}_{10}>14.3$ ). The model that outperformed the null model the most was the two main effects model (Baseline + rTMS condition: $\mathrm{BF}_{10}=287.7$ ). However, the data were only 1.5 times more likely under that model than under the model with the interaction $\left(\mathrm{BF}_{10}=187.3\right)$. We thus used planned comparisons to directly test the hypothesis that interference effects of STS stimulation mostly occurred in low baseline performers. These comparisons confirmed that, in lowperformers, active rTMS of STS increased IE scores (3.94 \pm 1.29$)$ compared to sham rTMS $(3.07 \pm$ 0.53; $P=0.003$; Cohens' $d=1.27)$ and active rTMS of V1 (3.30 $\pm 1.30 ; P=0.002$; Cohens' $d=1.36)$, which in turn did not differ from one another $(P=0.35$; Cohens' $d=0.34)$. In contrast, in high-performers, IE scores during active rTMS over STS (2.23 \pm 0.33$)$ were non-significantly greater than IE scores during sham rTMS $(1.93 \pm 0.27 ; P=0.24$; Cohens' $d=0.43)$ and active rTMS of V1 (1.96 $\pm 0.22 ; P=0.24$; Cohens' $d=0.56)$, which in turn did not differ from one another $(P=0.84$; Cohens' $d=0.05)$.

In Experiment 2, baseline performance did not predict performance changes following stimulation of IFG (Adjusted $R^{2}=0.03, F_{1,14}=1.45, P=0.19$ ) or TPJ (Adjusted $R^{2}=-0.03, F_{1,14}=0.52, P=$ $0.48)$. However, in Experiment 2, there was an outlier with standard residual $>2.5 \sigma$. After removal of the outlier, the regression model became marginally significant for the IFG condition and showed a medium/large effect size (Adjusted $R^{2}=0.20, F_{1,13}=4.46, P=0.054 ; f^{2}=0.34$ ), with low-performers showing the greatest impairment in performance following rTMS over IFG ( $\beta=$ $\left.0.51 ; t_{13}=2.11, P=0.054\right)$. Baseline performance was still a predictor of changes induced by IFGrTMS after controlling for the discomfort caused by IFG-rTMS $\left(\beta=0.55 ; t_{12}=2.19, P=0.049\right)$. Even more consistent results were observed in the TPJ condition, for which the regression model showed a large effect size (Adjusted $R^{2}=0.28, F_{1,13}=6.50, P=0.024 ; f^{2}=0.50$ ), with highperformers showing the greatest impairment in performance following rTMS over TPJ ( $\beta=-$ $\left.0.58, t_{13}=-2.55, P=0.024\right)$. Baseline performance remained a significant predictor after controlling for the discomfort caused by TPJ-rTMS $\left(\beta=-0.58 ; t_{12}=-2.39, P=0.034\right)$.

Fig. S1 shows an additional multiple regression analysis testing TPJ and IFG contributions (indexed by the interferential rTMS effects on IE scores) to efficient EA task performance during 
sham rTMS. Bayesian regression showed weak evidence in favor of the hypothesis that baseline performance predicts the performance change following IFG stimulation $\left(\mathrm{BF}_{10}=2.2\right)$ relative to the null model (including the effect of discomfort), whereas positive evidence was found in the case of the change following TPJ stimulation $\left(\mathrm{BF}_{10}=9.0\right)$.

These findings were corroborated by the Baseline (low-performers, high-performers) x rTMS condition (sham, IFG, TPJ) mixed factors ANOVA on IE scores. The analysis was performed after removing the outlier participant identified in the regression analysis. The ANOVA showed a main effect of Baseline $\left(F_{1,13}=8.61, P=0.012 ; \eta_{p}{ }^{2}=0.40\right)$, with high-performers showing lower IE scores than low-performers, and a main effect of rTMS condition $\left(F_{2,26}=10.19, P<0.001 ; \eta_{p}{ }^{2}=0.44\right)$, confirming the increase in IE scores following IFG and TPJ stimulation. Importantly, the Baseline x rTMS condition interaction was significant $\left(F_{2,26}=11.01, P<0.001 ; \eta_{p}{ }^{2}=0.46\right)$. Post-hoc analysis showed that, in low-performers, active rTMS of IFG increased IE scores $(3.50 \pm 0.51)$ compared to sham rTMS $(2.75 \pm 0.31 ; P=$ 0.002 ; Cohens' $d=1.47)$ and rTMS of TPJ $(2.79 \pm 0.21 ; P=0.003$; Cohens' $d=1.44)$ which in turn did not differ from one another $(P=0.61$; Cohens' $d=0.39)$. In high-performers, active rTMS of TPJ increased IE scores (3.08 \pm 0.58$)$ compared to sham rTMS $(2.05 \pm 0.19 ; P<0.001$; Cohens' $d=1.67)$ and rTMS of IFG $(2.43 \pm 0.52 ; P=0.033$; Cohens' $d=0.78)$ which in turn did not differ from one another $(P=0.10$; Cohens' $d=0.69)$.

In sum, across the two experiments, low-performers showed disrupted EA task performance when the STS and IFG were targeted, whereas they showed no interference when V1 or TPJ were targeted. In contrast, high-performers showed disrupted task performance only when the TPJ was stimulated, whereas they were not affected by stimulation of V1, STS or IFG. These findings suggest that STS, IFG and TPJ differentially contribute to the efficiency of EA task performance in low- and high-performers.

\section{Discussion}

Accurate understanding of the emotional feeling behind a facial expression (i.e., EA) is supported by multiple processes. Recent theoretical (Keysers and Gazzola, 2007, Uddin et al., 2007, Zaki et al., 2012a, Lamm and Majdandžić, 2015) and empirical work (Wheatley et al., 2007, Zaki et al., 2009b, Redcay et al., 2010, Schippers et al., 2010, Wolf et al., 2010, Lamm et al., 2011, Spunt and Lieberman, 2013, Raz et al., 2014) suggests that during complex social tasks, visual, sensorimotor and cognitive mechanisms might all be involved in understanding others. However, the relation between activity in brain regions supporting these mechanisms and participants' behavior is still unclear. This is because current knowledge is largely based on indirect correlational evidence. Building upon prior neuroimaging work suggesting that emotion perception and mental state 
inference are linked with neural activity in STS, IFG and TPJ (van Overwalle and Baetens, 2009, Mar, 2011, Sabatinelli et al., 2011, Bzdok et al., 2012, Grosbras et al., 2012, Molenberghs et al., 2012), this study sought to examine whether rTMS over these regions could affect the efficiency of EA, and whether individual differences in EA abilities could reveal differential causal contributions of STS, IFG and TPJ.

Our results showed that interference with STS (in Experiment 1) and interference with IFG and TPJ (in Experiment 2) reduced participants' efficiency in making explicit inferences about the level of enjoyment supposedly felt by smiling/laughing social targets (Zaki et al., 2009a, Zaki et al., 2009b, Harvey et al., 2013, Paracampo et al., 2017). This disruption in EA was indexed by a consistent increase in IE scores, ruling out that rTMS induced any criterion shift or speed-accuracy trade-off, in which participants achieved faster RTs by sacrificing accuracy or vice-versa. Changes in EA task efficiency were not due to unspecific effects of rTMS, as there was no relationship with the discomfort induced by rTMS and, critically, no behavioral interference was observed when stimulating a control site that induced similar discomfort as stimulation of the target areas. These results add to the growing literature suggesting that high-order visual, sensorimotor and mentalizing networks are co-activated and functionally coupled during social perception (Wheatley et al., 2007, Zaki et al., 2009b, Schippers et al., 2010, Harvey et al., 2013, Spunt and Lieberman, 2013, Raz et al., 2014) and showing that regions within these networks predict accurate performance in EA tasks requiring participants to evaluate the emotions supposedly felt by social targets (Zaki et al., 2009b, Harvey et al., 2013, McGettigan et al., 2015). Our findings expand these previous studies by establishing a more direct causal link between the target brain regions (STS, IFG and TPJ) and EA abilities.

An analysis of different behavioral parameters clarified the roles of STS, IFG and TPJ in EA task performance. rTMS over the three regions induced a consistent reduction in EA scores, indexing a weaker relationship between the perceiver's and the social targets' enjoyment ratings, and this effect was similarly large across the three regions. No effect was observed on raw enjoyment ratings, suggesting rTMS did not systematically increase or decrease such ratings but, rather, hindered their accuracy. rTMS over IFG significantly slowed down RTs in the EA task, whereas rTMS over TPJ and STS induced a marginally significant and a non-significant tendency for increased RTs, respectively. These findings indicate that STS, IFG and TPJ are all crucial for accurate inference of social targets' enjoyment from observed smiles and laughter. Moreover, IFG and, to a lesser extent, TPJ are required for providing faster EA judgments. Thus, our findings highlight particularly pivotal roles of neural regions supporting distinct sensorimotor and cognitive mechanisms in EA. 
Secondly, our study highlights the importance of individual differences in the neural bases of EA. Our exploratory analysis revealed that performance changes following rTMS over STS, IFG and TPJ were linearly related to individual differences in baseline EA task efficiency. The sign of the relationship varied across brain regions, with IFG and STS showing a positive relationship and TPJ showing a negative relationship. Splitting the sample into low- and high-performers in the baseline condition (i.e., during sham rTMS) showed that lower baseline performers were impaired when STS and IFG were targeted, but not when TPJ was targeted. In contrast, higher baseline performers showed impaired performance only when TPJ was targeted, and they were less affected by stimulation of STS or IFG. Adding to the specificity of our effects, we found no relationship between baseline EA task efficiency and performance changes following stimulation of the control site V1, and neither low-performers nor high-performers were affected by stimulation of this site. Collectively, the two experiments indicate that STS, IFG and TPJ are all functionally relevant to EA abilities; however, the double dissociation between low- and high-performers indicates that performance moderates the influence that these regions exert over EA abilities.

These findings are in line with recent accounts that highlight the important interplay between the outcomes of non-invasive brain stimulation and a variety of individual differences, such as gene polymorphisms, gender, age, personality and endogenous brain oscillations (Avenanti et al., 2009, Ridding and Ziemann, 2010, Bolognini et al., 2013, Hamada et al., 2013, Borgomaneri et al., 2015a, Borgomaneri et al., 2015b, Taschereau-Dumouchel et al., 2016, Bertossi et al., 2017).

Further studies have shown the importance of baseline performance on the degree of change in other cognitive abilities using different forms of non-invasive brain stimulation (Krause and Cohen $\underline{\text { Kadosh, 2014, Benwell et al., 2015, Painter et al., 2015, Emrich et al., 2017, Penton et al., }}$ 2017, Yang and Banissy, 2017).

Our findings suggest that STS, IFG and TPJ play different causal roles in supporting efficient EA abilities in low- and high-performers. Low-performers and high-performers may differentially recruit brain networks supporting EA abilities, with low-performing individuals relying more on STS and IFG, and high-performing individuals relying more on TPJ to infer enjoyment from observed smiles/laughter. This dissociation in the neural bases of EA suggests that optimal performance in our EA task might be mainly mediated by mentalizing mechanisms. When comparing TPJ and IFG contributions to efficient EA task performance in the baseline condition (indexed by the interferential rTMS effects on IE scores), interference with TPJ was the only significant predictor of baseline EA efficiency thus highlighting the unique contribution of TPJ in achieving efficient EA task performance (Fig. S1). These findings converge with and complement brain stimulation research showing that interfering with TPJ and other brain areas involved in mentalizing disrupts social and emotional judgments (Ferrari et al., 2016; Gamond and Cattaneo, 2016) and applying transcranial electrical stimulation (tES) aimed at increasing TPJ excitability 
improves performance in complex social tasks (Santiesteban et al., 2012, Donaldson et al., 2015, Hogeveen et al., 2015, Sowden et al., 2015). On the other hand, our data point to the possible constraints of applying tES to improve social cognitive functions, as they suggest that individual differences in baseline social abilities can moderate the effect of brain stimulation.

That the effect of IFG-rTMS was stronger in low-performers confirms and expands results from recent transcranial random noise stimulation (tRNS) studies (Penton et al., 2017, Yang and Banissy, 2017). In these studies, tRNS was administered to increase the excitability of the inferior frontal cortex before an emotion discrimination task. Interestingly, tRNS improved the efficiency of emotion discrimination, but only in participants with low baseline performance. Thus, despite several relevant differences between these studies and our rTMS study -including stimuli (static vs. dynamic face expressions), task (discrimination/categorization vs. evaluation of the intensity of the feeling) and induced changes in task efficiency (increase vs. decrease)- the results converge to indicate that individual differences in performance are an important moderator of the effect of IFG stimulation on efficient emotion processing.

It is also worth noting that we found similar results when targeting STS - a region that is thought to provide high-order visual representation of observed actions and emotional expressions to parietofrontal regions supporting sensorimotor simulation, including the IFG (Wood et al., 2016). Despite neuroimaging and neuropsychological meta-analytic studies highlighting a role for STS in action and emotion perception (Fusar-Poli et al., 2009, Sabatinelli et al., 2011, Urgesi et al., 2014), only some brain stimulation studies showed behavioral effects of STS stimulation (e.g., Grossman et al., 2005; Cattaneo et al., 2010a; Candidi et al., 2011; Pitcher, 2014; Makris and Urgesi, 2015), whereas other studies reported little or no effect (Pourtois et al., 2004, van Kemenade et al., 2012, Vonck et al., 2015, Mazzoni et al., 2017). Our finding that disruptive effects of STS-rTMS are mainly detected in low-performers rather than high performers suggests that individual differences and task difficulty might contribute to the variability in the outcome of STS stimulation seen in previous research.

The different findings observed with stimulation of STS, IFG and TPJ are in line with the notion that these regions provide distinct mechanisms for understanding others (Keysers and Gazzola, 2007, Uddin et al., 2007, Mitchell, 2009, van Overwalle and Baetens, 2009, Gallese and Sinigaglia, 2011, Lamm and Majdandžić, 2015) and with recent studies addressing the neural bases of EA abilities (Zaki et al., 2012b, Paracampo et al., 2017). In a neuroimaging study, Zaki et al. (2012b) scanned participants while they watched social targets discussing emotional autobiographical events. When participants performed an EA task requiring them to judge the social targets' emotional feelings, their emotion ratings predicted neural activity within the mentalizing network; when they performed a control task requiring them to judge targets' eye-gaze direction, recruitment of visual and sensorimotor areas was observed (Zaki et al., 2012b). Our study 
expands previous evidence by demonstrating a causal relationship between optimal EA task performance and TPJ involvement. Taken together, the study of Zaki et al. (2012b) and the present study suggest that, while visual and sensorimotor mechanisms might be recruited when seeing the (observable) emotional behavior of others, proficiently judging the intensity of the (unobservable) feelings behind that behavior critically relies on mentalizing mechanisms.

It is intriguing to note that in previous studies we have found that the same rTMS protocol used here, i.e., $6 \mathrm{~Hz}$ rTMS administered over IFG, but not over TPJ, disrupted performance in tasks requiring participants to discriminate between authentic and false expressions of enjoyment (Paracampo et al., 2017) or between deceptive and truthful actions (Tidoni et al., 2013), thus showing a role of sensorimotor networks, but not mentalizing networks, in social perception. In those studies, participants appeared to act similarly to the subgroup of low-performers in the present study. It is therefore possible that task difficulty and/or individual differences in baseline performance (as suggested by the present data) might explain the lack of any effect of TPJ stimulation. On the other hand, we should note that participants in the studies of Paracampo et al. (2017) and Tidoni et al. (2013) had to discriminate expressions/actions associated with fake/truthful feelings/intentions, and such discrimination could be performed by detecting body or facial signs that are diagnostic of fake actions/expressions. The mentalizing component was minimal compared with the present EA task that requires participants to explicitly and quantitatively estimate the intensity of the feeling behind observed smiles/laughter. It will thus be interesting for future work to combine different EA tasks requiring different levels of mental state inferences to further establish the moderators of brain network involvement in EA.

Understanding the reasons why low- and high-EA performers rely on partially different neural substrates is an important avenue for future work. It may reflect differences in spontaneous or strategic recruitment of visual, sensorimotor and cognitive mechanisms in EA (Meffert et al., $\underline{2013}$, Keysers and Gazzola, 2014) and such recruitment may vary as a function of task difficulty. Therefore, it will be interesting for future work to examine these questions, potentially by setting task difficulty for each individual participant. Also, individual differences in EA might reflect structural and functional differences, as has already been shown for simple emotion recognition tasks (Uono et al., 2016, Vicario et al., 2017) or empathy traits (Banissy et al., 2012, Eres et al., 2015); therefore, future work might combine structural and functional imaging with brain stimulation to address these questions.

It is important to consider the possibility that the relationship between baseline performance and performance changes following stimulation may reflect regression to the mean (Healy and Goldstein, 1978, Copas, 1997). The current findings are unlikely to be explained by this for three reasons. Firstly, high-performers showed the greatest performance change following TPJ stimulation. For regression to the mean to be likely, extreme low-performers would also have been 
expected to show an increase in task performance following stimulation, similar in magnitude to the decrease observed in high-performers. However, we did not observe such pattern. Secondly, while a negative relationship was observed between baseline IE scores and the interferential effect of TPJ stimulation, a similar trend was not observed for the other stimulation sites. If the results observed here were reflective of regression to the mean, a similar relationship would be expected regardless of the site of stimulation. Thirdly, instead of showing an improvement following active rTMS, low-performers showed even less efficient EA task performance when STS and IFG were targeted. These findings speak against a possible role of regression to the mean in accounting for our results.

\section{Conclusions}

In sum, the data presented here demonstrate that rTMS to STS, IFG and TPJ can affect the ability to efficiently infer the level of enjoyment from social targets' smiles/laughter, thus indicating that these regions play a critical role in EA. Moreover, we show that this effect is influenced by baseline EA task performance, suggesting critical roles of STS and IFG in low-performers and of TPJ in high-performers. Our study is consistent with imaging data suggesting differential roles of STS, IFG and TPJ in emotion understanding. It is also in line with emerging evidence from the field of brain stimulation research suggesting that individual differences play an important role in moderating the effects of stimulation. These findings may have theoretical implications for understanding the bases of EA abilities and for applied/clinical interventions in atypical populations, as they suggest that individual differences in EA might constrain the efficacy of brain stimulation in specific areas.

\section{Acknowledgments}

This work was supported by grants from the Ministero della Salute [Bando Ricerca Finalizzata Giovani Ricercatori 2010, grant number GR-2010-2319335], Cogito Foundation [Research project 2013, grant number R-117/13; and Research project 2014, grant number 14-139-R], Ministero Istruzione, Università e Ricerca [Futuro in Ricerca 2012, grant number RBFR12F0BD], Fondazione del Monte [399bis/2017] awarded to A.A., and by grants from the Bial Foundation [Grants Programme 2016/17, grant number 298/16] awarded to S.B. and A.A. We thank Brianna Beck for proofreading the manuscript. Author contributions: A.A. came up with the study concept, R.P., S.B., and A.A. designed the experiments; R.P., M.P., M.C. and S.B. performed the experiments; R.P., M.P. and A.A. analyzed the data; all authors wrote the manuscript. 


\section{References}

Allison, T., Puce, A., McCarthy, G., 2000. Social perception from visual cues: role of the STS regions. Trends Cogn. Sci. 4, 267-278.

Ambadar, Z., Cohn, J., Reed, L., 2009. All smiles are not created equal: morphology and timing of smiles perceived as amused, polite, and embarrassed/nervous. J. Nonverbal Behav. 33, 17-34.

Amodio, D.M., Frith, C.D., 2006. Meeting of minds: the medial frontal cortex and social cognition. Nat. Rev. Neurosci. 7, 268-277.

Avenanti, A., Annela, L., Serino, A., 2012. Suppression of premotor cortex disrupts motor coding of peripersonal space. Neuroimage 63, 281-288.

Avenanti, A., Annella, L., Candidi, M., Urgesi, C., Aglioti, S.M., 2013a. Compensatory plasticity in the action observation network: virtual lesions of STS enhance anticipatory simulation of seen actions. Cereb. Cortex 23, 570-580.

Avenanti, A., Candidi, M., Urgesi, C., 2013b. Vicarious motor activation during action perception: beyond correlational evidence. Front Hum. Neurosci. 7, 185.

Avenanti, A., Bolognini, N., Maravita, A., Aglioti, S.M., 2007. Somatic and motor components of action simulation. Curr. Biol. 17, 2129-2135.

Avenanti, A., Minio-Paluello, I., Bufalari, I., Aglioti, S.M., 2009. The pain of a model in the personality of an onlooker: influence of state-reactivity and personality traits on embodied empathy for pain. Neuroimage 44, 275-283.

Avenanti, A., Paracampo, R., Annella, L., Tidoni, E., Aglioti, S.M., 2017. Boosting and decreasing action prediction abilities through excitatory and inhibitory tDCS of inferior frontal cortex. Cereb. Cortex. http://dx.doi.org/10.1093/cercor/bhx041.

Banissy, M.J., Kanai, R., Walsh, V., Rees, G., 2012. Inter-individual differences in empathy are reflected in human brain structure. Neuroimage 62, 2034-2039.

Benwell, C.S.Y., Learmonth, G., Miniussi, C., Harvey, M., Thut, G., 2015. Non-linear effects of transcranial direct current stimulation as a function of individual baseline performance: evidence from biparietal tDCS influence on lateralized attention bias. Cortex 69, 152-165.

Bertossi, E., Peccenini, L., Solmi, A., Avenanti, A., Ciaramelli, E., 2017. Transcranial direct current stimulation of the medial prefrontal cortex dampens mind-wandering in men. Sci. Rep. 7, 16962.

Bolognini, N., Rossetti, A., Convento, S., Vallar, G., 2013. Understanding others' feelings: the role of the right primary somatosensory cortex in encoding the affective valence of others' touch. J. Neurosci. 33, 4201-4205.

Borgomaneri, S., Gazzola, V., Avenanti, A., 2012. Motor mapping of implied actions during perception of emotional body language. Brain Stimul. 5, 70-76.

Borgomaneri, S., Gazzola, V., Avenanti, A., 2015a. Transcranial magnetic stimulation reveals two functionally distinct stages of motor cortex involvement during perception of emotional body language. Brain Struct. Funct. 220, 2765-2781.

Borgomaneri, S., Vitale, F., Gazzola, V., Avenanti, A., 2015b. Seeing fearful body language rapidly freezes the observer's motor cortex. Cortex 65, 232-245.

Bzdok, D., Schilbach, L., Vogeley, K., Schneider, K., Laird, A.R., Langner, R., Eickhoff, S.B., 2012. Parsing the neural correlates of moral cognition: ALE meta-analysis on morality, theory of mind, and empathy. Brain Struct. Funct. 217, 783-796.

Calvo, M.G., Nummenmaa, L., 2016. Perceptual and affective mechanisms in facial expression recognition: an integrative review. Cogn. Emot. 30, 1081-1106.

Candidi, M., Stienen, B.M.C., Aglioti, S.M., de Gelder, B., 2011. Event-related repetitive transcranial magnetic stimulation of posterior superior temporal sulcus improves the detection of threatening postural changes in human bodies. J. Neurosci. 31, 17547-17554.

Carducci, F., Brusco, R., 2012. Accuracy of an individualized MR-based head model for navigated brain stimulation. Psychiatry Res. 203, 105-108.

Carlin, J.D., Calder, A.J., 2013. The neural basis of eye gaze processing. Curr. Opin. Neurobiol. 23, $450-455$. 
Carr, L., Iacoboni, M., Dubeau, M.-C., Mazziotta, J.C., Lenzi, G.L., 2003. Neural mechanisms of empathy in humans: a relay from neural systems for imitation to limbic areas. Proc. Natl. Acad. Sci. USA 100, 5497-5502.

Cashdan, E., 1998. Smiles, speech, and body posture: how women and men display sociometric status and power. J. Nonverbal Behav. 22, 209-229.

Caspers, S., Zilles, K., Laird, A.R., Eickhoff, S.B., 2010. ALE meta-analysis of action observation and imitation in the human brain. Neuroimage 50, 1148-1167.

Cattaneo, L., Sandrini, M., Schwarzbach, J., 2010. State-dependent TMS reveals a hierarchical representation of observed acts in the temporal, parietal, and premotor cortices. Cereb. Cortex 20, 2252-2258.

Cohen, J., 1992. A power primer. Psychol. Bull. 112, 155-159.

Copas, J.B., 1997. Using regression models for prediction: shrinkage and regression to the mean. Stat. Methods Med. Res. 6, 167-183.

Costa, A., Torriero, S., Oliveri, M., Caltagirone, C., 2008. Prefrontal and temporo-parietal involvement in taking others' perspective: TMS evidence. Behav. Neurol. 19, 71-74.

Dapretto, M., Davies, M., Pfeifer, J., 2006. Understanding emotions in others: mirror neuron dysfunction in children with autism spectrum disorders. Nat. Neurosci. 9, 28-30.

Davis, M.H., 1983. Measuring individual differences in empathy: evidence for a multidimensional approach. J. Pers. Soc. Psychol. 44, 113-126.

Davis, M.H., 1996. Empathy: a Social Psychological Approach. Avalon Publishing.

Dienes, Z., 2011. Bayesian versus orthodox statistics: which side are you on? Perspect. Psychol. Sci. 6, 274-290.

Donaldson, P.H., Rinehart, N.J., Enticott, P.G., 2015. Noninvasive stimulation of the temporoparietal junction: a systematic review. Neurosci. Biobehav Rev. 55, 547-572.

Dunlap, W., Cortina, J., Vaslow, J., Burke, M., 1996. Meta-analysis of experiments with matched groups or repeated measures designs. Psychol. Methods 1, 170-177.

Ekman, P., Friesen, W., 1982. Felt, false, and miserable smiles. J. Nonverbal Behav. 6, 238-252.

Emrich, S.M., Johnson, J.S., Sutterer, D.W., Postle, B.R., 2017. Comparing the effects of $10-\mathrm{Hz}$ repetitive TMS on tasks of visual STM and attention. J. Cogn. Neurosci. 29, 286-297.

Eres, R., Decety, J., Louis, W.R., Molenberghs, P., 2015. Individual differences in local gray matter density are associated with differences in affective and cognitive empathy. Neuroimage 117, 305-310.

Ferrari, C., Lega, C., Vernice, M., Tamietto, M., Mende-Siedlecki, P., Vecchi, T., Todorov, A., Cattaneo, Z., 2016. The dorsomedial prefrontal cortex plays a causal role in integrating social impressions from faces and verbal descriptions. Cereb. Cortex 26, 156-165.

Frank, M., Ekman, P., 1993. Not all smiles are created equal: the differences between enjoyment and nonenjoyment smiles. Humor Int. J. Humor Res. 6, 9-26.

Fridlund, A., 2002. The behavioral ecology view of smiling and other facial expressions. In: Millicent, H.A. (Ed.), An Empirical Reflection on the Smile. Edwin Mellen Press.

Frith, C.D., Frith, U., 2006. The neural basis of mentalizing. Neuron 50, 531-534.

Fusar-Poli, P., Placentino, A., Carletti, F., Landi, P., Allen, P., Surguladze, S., Benedetti, F., Abbamonte, M., Gasparotti, R., Barale, F., Perez, J., McGuire, P., Politi, P., 2009. Functional atlas of emotional faces processing: a voxel-based meta-analysis of 105 functional magnetic resonance imaging studies. J. Psychiatry Neurosci. 34, 418-432.

Gallese, V., Gernsbacher, M.A., Heyes, C., Hickok, G., Iacoboni, M., 2011. Mirror neuron forum. Perspect. Psychol. Sci. 6, 369-407.

Gallese, V., Sinigaglia, C., 2011. What is so special about embodied simulation? Trends Cogn. Sci. $15,512-519$.

Gamond, L., Cattaneo, Z., 2016. The dorsomedial prefrontal cortex plays a causal role in mediating in-group advantage in emotion recognition: a TMS study. Neuropsychologia 93, 312-317.

Goldman, A.I., Sripada, C.S., 2005. Simulationist models of face-based emotion recognition. Cognition 94, 193-213.

Grosbras, M.H., Beaton, S., Eickhoff, S.B., 2012. Brain regions involved in human movement perception: a quantitative voxel-based meta-analysis. Hum. Brain Mapp. 33, 431-454. 
Grossman, E.D., Battelli, L., Pascual-Leone, A., 2005. Repetitive TMS over posterior STS disrupts perception of biological motion. Vision. Res. 45, 2847-2853.

Hamada, M., Murase, N., Hasan, A., Balaratnam, M., Rothwell, J.C., 2013. The role of interneuron networks in driving human motor cortical plasticity. Cereb. Cortex 23, 1593-1605.

Harris, R.J., Young, A.W., Andrews, T.J., 2012. Morphing between expressions dissociates continuous from categorical representations of facial expression in the human brain. Proc. Natl. Acad. Sci. USA 109, 21164-21169.

Harvey, P.O., Zaki, J., Lee, J., Ochsner, K., Green, M.F., 2013. Neural substrates of empathic accuracy in people with schizophrenia. Schizophr. Bull. 39, 617-628.

Haxby, J.V., Hoffman, E. a., Gobbini, M.I., 2000. The distributed human neural system for face perception. Trends Cogn. Sci. 4, 223-233.

Healy, M.J., Goldstein, H., 1978. Regression to the mean. Ann. Hum. Biol. 5, 277-280.

Hess, U., Kleck, R.E., 1994. The cues decoders use in attempting to differentiate emotion elicited and posed facial expressions. Eur. J. Soc. Psychol. 24, 367-381.

Hogeveen, J., Obhi, S.S., Banissy, M.J., Santiesteban, I., Press, C., Catmur, C., Bird, G., 2015. Task-dependent and distinct roles of the temporoparietal junction and inferior frontal cortex in the control of imitation. Soc. Cogn. Affect Neurosci. 10, 1003-1009.

Ickes, W., 1997. In: Press, G. (Ed.), Empathic Accuracy. The Guilford Press, New York.

Ickes, W., Stinson, L., 1990. Naturalistic social cognition: empathic accuracy in mixed-sex dyads. J. Pers. Soc. Psychol. 59, 730-742.

Jacquet, P.O., Avenanti, A., 2015. Perturbing the action observation network during perception and categorization of actions' goals and grips: state-dependency and virtual lesion TMS effects. Cereb. Cortex 598-608.

Jarosz, A.F., Wiley, J., 2014. What are the odds? a practical guide to computing and reporting bayes factors? J. Prob. Solving 7(2).

Jasp Team, 2017. Jasp (Version 0.8.5) [Computer Software].

Jellema, T., Perrett, D.I., 2006. Neural representations of perceived bodily actions using a categorical frame of reference. Neuropsychologia 44, 1535-1546.

Johnston, P., Mayes, A., Hughes, M., Young, A.W., 2013. Brain networks subserving the evaluation of static and dynamic facial expressions. Cortex 49, 2462-2472.

Jones, C.B., Lulic, T., Bailey, A.Z., Mackenzie, T.N., Mi, Y.Q., Tommerdahl, M., Nelson, A.J., 2016. Metaplasticity in human primary somatosensory cortex: effects on physiology and tactile perception. J. Neurophysiol. 115, 2681-2691.

Keysers, C., Gazzola, V., 2007. Integrating simulation and theory of mind: from self to social cognition. Trends Cogn. Sci. 11, 194-196.

Keysers, C., Gazzola, V., 2014. Dissociating the ability and propensity for empathy. Trends Cogn. Sci. $18,163-166$.

Koivisto, M., Mäntylä, T., Silvanto, J., 2010. The role of early visual cortex (V1/V2) in conscious and unconscious visual perception. Neuroimage 51, 828-834.

Korb, S., Grandjean, D., Scherer, K.R., 2010. Timing and voluntary suppression of facial mimicry to smiling faces in a Go/NoGo task-an EMG study. Biol. Psychol. 85, 347-349.

Korb, S., With, S., Niedenthal, P., Kaiser, S., Grandjean, D., 2014. The perception and mimicry of facial movements predict judgments of smile authenticity. PLoS One 9, e99194.

Korb, S., Malsert, J., Rochas, V., Rihs, T.A., Rieger, S.W., Schwab, S., Niedenthal, P.M., Grandjean, D., 2015. Gender differences in the neural network of facial mimicry of smiles-An rTMS study. Cortex 70, 101-114.

Krause, B., Cohen Kadosh, R., 2014. Not all brains are created equal: the relevance of individual differences in responsiveness to transcranial electrical stimulation. Front Syst. Neurosci. 8, 25.

Lamm, C., Decety, J., Singer, T., 2011. Meta-analytic evidence for common and distinct neural networks associated with directly experienced pain and empathy for pain. Neuroimage 54, 2492-2502.

Lamm, C., Majdandžić, J., 2015. The role of shared neural activations, mirror neurons, and morality in empathy - a critical comment. Neurosci. Res. 90, 15-24. 
Levenson, R.W., Ruef, A.M., 1992. Empathy: a physiological substrate. J. Pers. Soc. Psychol. 63, 234-246.

Makris, S., Urgesi, C., 2015. Neural underpinnings of superior action prediction abilities in soccer players. Soc. Cogn. Affect Neurosci. 10, 342-351.

Mar, R.A., 2011. The neural bases of social cognition and story comprehension. Annu Rev. Psychol. 62, 103-134.

Mazzoni, N., Jacobs, C., Venuti, P., Silvanto, J., Cattaneo, L., 2017. State-Dependent TMS Reveals Representation of Affective Body Movements in the Anterior Intraparietal Cortex. J. Neurosci. 37, 7231-7239.

McGettigan, C., Walsh, E., Jessop, R., Agnew, Z.K., Sauter, D. a., Warren, J.E., Scott, S.K., 2015. Individual differences in laughter perception reveal roles for mentalizing and sensorimotor systems in the evaluation of emotional authenticity. Cereb. Cortex 246-257.

McLellan, T., Johnston, L., Dalrymple-Alford, J., Porter, R., 2010. Sensitivity to genuine versus posed emotion specified in facial displays. Cogn. Emot. 24, 1277-1292.

Meffert, H., Gazzola, V., Den Boer, J.A., Bartels, A. a.J., Keysers, C., 2013. Reduced spontaneous but relatively normal deliberate vicarious representations in psychopathy. Brain 136, 25502562.

Mitchell, J.P., 2009. Inferences about mental states. Philos. Trans. R. Soc. Lond. B Biol. Sci. 364, 1309-1316.

Molenberghs, P., Cunnington, R., Mattingley, J.B., 2012. Brain regions with mirror properties: a meta-analysis of 125 human fMRI studies. Neurosci. Biobehav Rev. 36, 341-349.

Morris, S.B., DeShon, R.P., 2002. Combining effect size estimates in meta-analysis with repeated measures and independent-groups designs. Psychol. Methods 7, 105-125.

Niedenthal, P.M., Mermillod, M., Maringer, M., Hess, U., 2010. The Simulation of Smiles (SIMS) model: embodied simulation and the meaning of facial expression. Behav. Brain Sci. 33, 417$33-80$.

Nummenmaa, L., Calder, A.J., 2009. Neural mechanisms of social attention. Trends Cogn. Sci. 13, $135-143$.

Oberman, L.M., Winkielman, P., Ramachandran, V.S., 2007. Face to face: blocking facial mimicry can selectively impair recognition of emotional expressions. Soc. Neurosci. 2, 167-178.

Painter, D.R., Dux, P.E., Mattingley, J.B., 2015. Distinct roles of the intraparietal sulcus and temporoparietal junction in attentional capture from distractor features: an individual differences approach. Neuropsychologia 74, 50-62.

Palmer, C.E., Bunday, K.L., Davare, M., Kilner, J.M., 2016. A causal role for primary motor cortex in perception of observed actions. J. Cogn. Neurosci. 28, 2021-2029.

Paracampo, R., Tidoni, E., Borgomaneri, S., di Pellegrino, G., Avenanti, A., 2017. Sensorimotor network crucial for inferring amusement from smiles. Cereb. Cortex 27, 5116-5129.

Penton, T., Dixon, L., Evans, L., Banissy, M.J., 2017. Emotion perception improvement following transcranial random noise of the inferior frontal cortex. Sci. Rep. 7, 11278.

Pernigo, S., Moro, V., Avesani, R., Miatello, C., Urgesi, C., Aglioti, S.M., 2012. Massive somatic deafferentation and motor deefferentation of the lower part of the body impair its visual recognition: a psychophysical study of patients with spinal cord injury. Eur. J. Neurosci. 36, 3509-3518.

Pitcher, D., 2014. Facial expression recognition takes longer in the posterior superior temporal sulcus than in the occipital face area. J. Neurosci. 34, 9173-9177.

Pobric, G., Hamilton, A.F., 2006. Action understanding requires the left inferior frontal cortex. Curr. Biol. 16, 524-529.

Pourtois, G., Sander, D., Andres, M., Grandjean, D., Reveret, L., Olivier, E., Vuilleumier, P., 2004. Dissociable roles of the human somatosensory and superior temporal cortices for processing social face signals. Eur. J. Neurosci. 20, 3507-3515.

Pourtois, G., Schettino, A., Vuilleumier, P., 2013. Brain mechanisms for emotional influences on perception and attention: what is magic and what is not. Biol. Psychol. 92, 492-512.

Raftery, A., 1995. Bayesian model selection in social research. Sociol. Methodol. 25, 111-196. 
Raz, G., Jacob, Y., Gonen, T., Winetraub, Y., Flash, T., Soreq, E., Hendler, T., 2014. Cry for her or cry with her: context-dependent dissociation of two modes of cinematic empathy reflected in network cohesion dynamics. Soc. Cogn. Affect Neurosci. 9, 30-38.

Redcay, E., Dodell-Feder, D., Pearrow, M.J., Mavros, P.L., Kleiner, M., Gabrieli, J.D.E., Saxe, R., 2010. Live face-to-face interaction during fMRI: a new tool for social cognitive neuroscience. Neuroimage 50, 1639-1647.

Ridding, M.C., Ziemann, U., 2010. Determinants of the induction of cortical plasticity by noninvasive brain stimulation in healthy subjects. J. Physiol. 588, 2291-2304.

Rizzolatti, G., Cattaneo, L., Fabbri-Destro, M., Rozzi, S., 2014. Cortical mechanisms underlying the organization of goal-directed actions and mirror neuron-based action understanding. Physiol. Rev. 94, 655-706.

Romei, V., Chiappini, E., Hibbard, P.B., Avenanti, A., 2016. Empowering reentrant projections from V5 to V1 boosts sensitivity to motion. Curr. Biol. 26, 2155-2160.

Rossi, S., Hallett, M., Rossini, P.M., Pascual-Leone, A., 2009. Safety, ethical considerations, and application guidelines for the use of transcranial magnetic stimulation in clinical practice and research. Clin. Neurophysiol. 120, 2008-2039.

Rossini, P.M., et al., 2015. Non-invasive electrical and magnetic stimulation of the brain, spinal cord, roots and peripheral nerves: basic principles and procedures for routine clinical and research application. An updated report from an I.F.C.N. Committee. Clin. Neurophysiol. 126, 1071-1107.

Rychlowska, M., Cañadas, E., Wood, A., Krumhuber, E.G., Fischer, A., Niedenthal, P.M., 2014. Blocking mimicry makes true and false smiles look the same. PLoS One 9, e90876.

Saarimäki, H., Gotsopoulos, A., Jääskeläinen, I.P., Lampinen, J., Vuilleumier, P., Hari, R., Sams, M., Nummenmaa, L., 2016. Discrete neural signatures of basic emotions. Cereb. Cortex 26, 2563-2573.

Sabatinelli, D., Fortune, E.E., Li, Q., Siddiqui, A., Krafft, C., Oliver, W.T., Beck, S., Jeffries, J., 2011. Emotional perception: meta-analyses of face and natural scene processing. Neuroimage $54,2524-2533$.

Santiesteban, I., Banissy, M.J., Catmur, C., Bird, G., 2012. Enhancing social ability by stimulating right temporoparietal junction. Curr. Biol. 22, 2274-2277.

Saxe, R., 2006. Uniquely human social cognition. Curr. Opin. Neurobiol. 16, 235-239.

Schippers, M.B., Roebroeck, A., Renken, R., Nanetti, L., Keysers, C., 2010. Mapping the information flow from one brain to another during gestural communication. Proc. Natl. Acad. Sci. USA 107, 9388-9393.

Schurz, M., Radua, J., Aichhorn, M., Richlan, F., Perner, J., 2014. Fractionating theory of mind: a meta-analysis of functional brain imaging studies. Neurosci. Biobehav Rev. 42, 9-34.

Silvanto, J., Cowey, A., Lavie, N., Walsh, V., 2005. Striate cortex (V1) activity gates awareness of motion. Nat. Neurosci. 8, 143-144.

Sowden, S., Wright, G.R.T., Banissy, M.J., Catmur, C., Bird, G., 2015. Transcranial current stimulation of the temporoparietal junction improves lie detection. Curr. Biol. 25, 2447-2451.

Spunt, R.P.R., Lieberman, M.D., 2013. The busy social brain evidence for automaticity and control in the neural systems supporting social cognition and action understanding. Psychol. Sci. 24, $80-86$.

Stuart, A., Ord, J.K., 1987. Kendall's Advanced Theory of Statistics, Vol. 1: Distribution Theory. 1 Charles Griffin, London.

Taschereau-Dumouchel, V., Hétu, S., Michon, P.-E., Vachon-Presseau, E., Massicotte, E., De Beaumont, L., Fecteau, S., Poirier, J., Mercier, C., Chagnon, Y.C., Jackson, P.L., 2016. BDNF Val66Met polymorphism influences visuomotor associative learning and the sensitivity to action observation. Sci. Rep. 6, 34907.

Tidoni, E., Borgomaneri, S., di Pellegrino, G., Avenanti, A., 2013. Action simulation plays a critical role in deceptive action recognition. J. Neurosci. 33, 611-623.

Townsend, J., Ashby, F., 1983. Stochastic Modeling of Elementary Psychological Processes. Cambridge University Press, Cambridge. 
Uddin, L.Q., Iacoboni, M., Lange, C., Keenan, J.P., 2007. The self and social cognition: the role of cortical midline structures and mirror neurons. Trends Cogn. Sci. 11, 153-157.

Uono, S., Sato, W., Kochiyama, T., Sawada, R., Kubota, Y., Yoshimura, S., Toichi, M., 2016. Neural substrates of the ability to recognize facial expressions: a voxel-based morphometry study. Soc. Cogn. Affect Neurosci. 12, 487-495.

Urgesi, C., Berlucchi, G., Aglioti, S.M., 2004. Magnetic stimulation of extrastriate body area impairs visual processing of nonfacial body parts. Curr. Biol. 14, 2130-2134.

Urgesi, C., Calvo-Merino, B., Haggard, P., Aglioti, S.M., 2007. Transcranial magnetic stimulation reveals two cortical pathways for visual body processing. J. Neurosci. 27, 8023-8030.

Urgesi, C., Candidi, M., Avenanti, A., 2014. Neuroanatomical substrates of action perception and understanding: an anatomic likelihood estimation meta-analysis of lesion-symptom mapping studies in brain injured patients. Front Hum. Neurosci. 8, 344.

Valchev, N., Tidoni, E., Hamilton, A.F., Gazzola, V., Avenanti, A., 2017. Primary Somatosensory cortex necessary for the perception of weight from other people's action: a continuous thetaburst TMS experiment. Neuroimage 152, 195-206.

van Kemenade, B.M., Muggleton, N., Walsh, V., Saygin, A.P., 2012. Effects of TMS over premotor and superior temporal cortices on biological motion perception. J. Cogn.Neurosci. 24, 896-904.

van Overwalle, F., Baetens, K., 2009. Understanding others' actions and goals by mirrorand mentalizing systems: a meta-analysis. Neuroimage 48, 564-584.

Vicario, C.M., Rafal, R.D., Martino, D., Avenanti, A., 2017. Core, social and moral disgust are bounded: a review on behavioral and neural bases of repugnance in clinical disorders. Neurosci. Biobehav. Rev. 80, 185-200.

Vonck, S., Swinnen, S.P., Wenderoth, N., Alaerts, K., 2015. Effects of transcranial direct current stimulation on the recognition of bodily emotions from point-light displays. Front Hum. Neurosci. 9, 438.

Vuilleumier, P., Huang, Y.-M., 2009. Emotional attention. Curr. Dir. Psychol. Sci. 18, 148-152.

Wagenmakers, E.-J., Marsman, M., Jamil, T., Ly, A., Verhagen, J., Love, J., Selker, R., Gronau, Q.F., Šmíra, M., Epskamp, S., Matzke, D., Rouder, J.N., Morey, R.D., 2017. Bayesian inference for psychology. Part I: theoretical advantages and practical ramifications. Psychon. Bull. Rev. $1-23$.

Wheatley, T., Milleville, S.C., Martin, A., 2007. Understanding animate agents: distinct roles for the social network and mirror system. Psychol. Sci. 18, 469-474.

Winston, J., O’Doherty, J., Dolan, R., 2003. Common and distinct neural responses during direct and incidental processing of multiple facial emotions. Neuroimage 20, 84-97.

Winston, J.S., Henson, R.N.A., Fine-Goulden, M.R., Dolan, R.J., 2004. fMRI-adaptation reveals dissociable neural representations of identity and expression in face perception. J. Neurophysiol. 92, 1830-1839.

Wolf, I., Dziobek, I., Heekeren, H.R., 2010. Neural correlates of social cognition in naturalistic settings: a model-free analysis approach. Neuroimage 49, 894-904.

Wood, A., Rychlowska, M., Korb, S., Niedenthal, P., 2016. Fashioning the face: sensorimotor simulation contributes to facial expression recognition. Trends Cogn. Sci. 20, 227-240.

World Health Organisation, 2013. Declaration of helsinki world medical association declaration of Helsinki ethical principles for medical research involving human subjects. J. Am. Med Assoc. 310, 2191-2194.

Yang, T., Banissy, M.J., 2017. Enhancing anger perception in older adults by stimulating inferior frontal cortex with high frequency transcranial random noise stimulation. Neuropsychologia 102, 163-169.

Young, L., Camprodon, J.A., Hauser, M., Pascual-Leone, A., Saxe, R., 2010. Disruption of the right temporoparietal junction with transcranial magnetic stimulation reduces the role of beliefs in moral judgments. Proc. Natl. Acad. Sci. USA 107, 6753-6758.

Zaki, J., Bolger, N., Ochsner, K., 2008. It Takes two the interpersonal nature of empathic accuracy. Psychol. Sci. 19, 399-404. 
Zaki, J., Bolger, N., Ochsner, K., 2009a. Unpacking the informational bases of empathic accuracy. Emotion 9, 478-487.

Zaki, J., Ochsner, K.N., Ochsner, K., 2012a. The neuroscience of empathy: progress, pitfalls and promise. Nat. Neurosci. 15, 675-680.

Zaki, J., Weber, J., Bolger, N., Ochsner, K.N., 2009b. The neural bases of empathic accuracy. Proc. Natl. Acad. Sci. USA 106, 11382-11387.

Zaki, J., Weber, J., Ochsner, K., 2012b. Task-dependent neural bases of perceiving emotionally expressive targets. Front Hum. Neurosci. 6, 228. 